\title{
REES ALGEBRAS OF ADDITIVE GROUP ACTIONS
}

\author{
ADRIEN DUBOULOZ, ISAC HEDÉN, AND TAKASHI KISHIMOTO
}

\begin{abstract}
We establish basic properties of a sheaf of graded algebras canonically associated to every relative affine scheme $f: X \rightarrow S$ endowed with an action of the additive group scheme $\mathbb{G}_{a, S}$ over a base scheme or algebraic space $S$, which we call the (relative) Rees algebra of the $\mathbb{G}_{a, S}$-action. We illustrate these properties on several examples which played important roles in the development of the algebraic theory of locally nilpotent derivations and give some applications to the construction of families of affine threefolds with $\mathbb{G}_{a}$-actions.
\end{abstract}

\section{INTRODUCTION}

The study of regular actions of the additive group $\mathbb{G}_{a}$ on affine varieties has led to an increased understanding of both algebraic and geometric properties of these varieties. A $\mathbb{G}_{a}$-action on an affine variety $X$ defined over a field $k$ of characteristic zero is fully determined by its velocity vector field, which takes the form of a $k$-derivation $\partial$ of the coordinate ring $A$ of $X$ with the property that $A$ is the increasing union of the kernels of the iterated $k$-linear differential operators $\partial^{n}, n \geq 1$. Due to this correspondence, the study of regular $\mathbb{G}_{a}$-actions developed into a very rich algebraic theory of such differential operators, called locally nilpotent $k$-derivations. The most fundamental object associated to such a derivation is its kernel, which coincides with the subalgebra of $\mathbb{G}_{a}$-invariant functions on $X$. Kernels of locally nilpotent derivations have been intensively studied during the last decades, with many applications to the construction of new invariants to distinguish affine spaces among all affine varieties and to the understanding of automorphism groups of affine varieties close to affine spaces (see [15] and the references therein). A second natural subspace associated to a locally nilpotent $k$-derivation $\partial$ of a $k$-algebra $A$ which has been very much studied from an algebraic point of view is the kernel Ker $\partial^{2}$ of its square $\partial^{2}$. Geometrically, the elements of Ker $\partial^{2} \backslash$ Ker $\partial$, usually called local slices, are regular functions on $X=\operatorname{Spec}(A)$ which restrict to coordinate functions on general orbits of the corresponding $\mathbb{G}_{a}$-action. The image of $\left.\partial\right|_{\operatorname{Ker}^{2}}: \operatorname{Ker} \partial^{2} \rightarrow \operatorname{Ker} \partial$ is an ideal of Ker $\partial$, called the plinth ideal, which encodes basic geometric properties of the algebraic quotient morphism $X \rightarrow X / / \mathbb{G}_{a}=\operatorname{Spec}(\operatorname{Ker} \partial)$.

A more systematic study of the algebro-geometric properties encoded by the whole increasing exhaustive filtration of $A$ formed by the subspaces $F_{n}=\operatorname{Ker}^{n+1}, n \geq 0$, was initiated only quite recently by Alhajjar $[1,2]$ and Freudenburg [14]. For instance, they observed that for an integral finitely generated $k$-algebra $A$ endowed with a nonzero locally nilpotent $k$-derivation $\partial$, the infinite collection of inclusions $F_{n} \hookrightarrow F_{n+1}$, $n \geq 0$, gives rise to a collection of successive inclusions $k\left[F_{n}\right] \hookrightarrow k\left[F_{n+1}\right]$ between the $k$-subalgebras of $A$ that they generate. This sequence exhausts $A$ after finitely many steps, i.e. $k\left[F_{r}\right]=A$ for some $r \in \mathbb{N}$. These inclusions correspond geometrically to a canonical sequence of birational $\mathbb{G}_{a}$-equivariant morphisms

$$
X=\operatorname{Spec}(A) \cong \operatorname{Spec}\left(k\left[F_{r}\right]\right) \rightarrow \operatorname{Spec}\left(k\left[F_{r-1}\right]\right) \rightarrow \cdots \rightarrow \operatorname{Spec}\left(k\left[F_{1}\right]\right)
$$

factorizing the algebraic quotient morphism $X \rightarrow \operatorname{Spec}\left(F_{0}\right)$. The basic properties of this factorization have been established by Freudenburg [14]. He described in particular an algorithm to compute, under suitable noetherianity conditions, the subspaces $F_{n}, n \geq 0$, as well as the corresponding algebras $k\left[F_{n}\right]$.

2000 Mathematics Subject Classification. 14R20; 14R25; 14L30; 13A30.

The first author was partially supported by the French "Investissements d'Avenir" program, project ISITE-BFC (contract ANR-IS-IDEXOOOB) and from ANR Project FIBALGA ANR-18-CE40-0003-01. The second author gratefully acknowledges support from the Knut and Alice Wallenberg Foundation, grant number KAW2016.0438. The third author was partially funded by Grant-in-Aid for Scientific Research of JSPS No. 15K04805 and No. 19K03395. The authors thank the University of Saitama, at which this research was initiated during visits of the first and second authors, and the Institute of Mathematics of Burgundy, at which it was continued during a visit of the third author, for their generous support and the excellent working conditions offered. 
In this article, we shift the focus to a complementary approach which considers the properties of the two natural graded algebras that can be canonically associated to a filtered algebra: its associated graded algebra and its Rees algebra. For a $k$-algebra $A$ with a nonzero locally nilpotent $k$-derivation $\partial$, these are thus the algebras

$$
\operatorname{gr}_{\partial} A=\bigoplus_{n \geq 0} \operatorname{Ker}^{n+1} / \operatorname{Ker}^{n} \quad \text { and } \quad R(A, \partial)=\bigoplus_{n \geq 0} \operatorname{Ker}^{n+1} .
$$

The first one already plays an important role in the computation of Makar-Limanov invariants of certain affine varieties [22, 2], but to our knowledge, the second one, which we henceforth call the Rees algebra of $(A, \partial)$, has not been considered before in this context. Besides the fact that these Rees algebras are functorial with respect to $\mathbb{G}_{a}$-equivariant morphisms, two basic properties which motivate their study are the following:

- First, the canonical graded homomorphism of degree 0

$$
k[\theta]=\bigoplus_{n \geq 0} k \rightarrow \bigoplus_{n \geq 0} \operatorname{Ker}^{n+1}
$$

induced by the inclusion $k \subset \operatorname{Ker} \partial^{n+1}$ for every $n \geq 0$ provides a one-parameter deformation

$$
\pi: \operatorname{Spec}(R(A, \partial)) \rightarrow \mathbb{A}_{k}^{1}=\operatorname{Spec}(k[\theta])
$$

whose fibers over the points 0 and 1 are canonically isomorphic to $\operatorname{Spec}\left(\operatorname{gr}_{\partial} A\right)$ and $\operatorname{Spec}(A)$ respectively.

- Second, the Rees algebra $R(A, \partial)$ carries canonical extensions of $\partial$ to homogeneous locally nilpotent $k[\theta]$-derivations of homogeneous degree $m$ for every $m \geq-1$, whose corresponding $\mathbb{G}_{a}$-actions on $\operatorname{Spec}(R(A, \partial))$ make $\pi$ a $\mathbb{G}_{a}$-equivariant deformation of $\operatorname{Spec}(A)$ endowed with the $\mathbb{G}_{a}$-action defined by $\partial$. For $m=-1$, the induced $\mathbb{G}_{a}$-action on the fiber $\pi^{-1}(0)$ coincides with the one which is defined by the homogeneous $k$-derivation gr $\partial$ of $\operatorname{gr}_{\partial} A$ of degree -1 , the latter derivation being canonically associated to $\partial$. On the other hand, for $m=0$ the $\mathbb{G}_{a}$-action on $\operatorname{Spec}(R(A, \partial))$ descends to a $\mathbb{G}_{a}$-action on $\operatorname{Proj}(R(A, \partial))$ and we obtain in particular a canonical $\mathbb{G}_{a}$-equivariant open embedding $\operatorname{Spec}(A) \hookrightarrow \operatorname{Proj}(R(A, \partial))$ which provides a canonical relative $\mathbb{G}_{a}$-equivariant completion of $\operatorname{Spec}(A)$ over $\operatorname{Spec}(\operatorname{Ker} \partial)$.

Example. The Rees algebra $R\left(k[t], \frac{\partial}{\partial t}\right)$ of the additive group $\mathbb{G}_{a}$ acting on itself by translations is isomorphic to the polynomial ring in two variables $k[t, \theta]$ with its standard grading, and the associated graded algebra gr $\frac{\partial}{\partial t} k[t]$ is the the polynomial ring $k[t]$ endowed with its standard grading. The locally nilpotent $k[\theta]-$ derivation $\frac{\partial}{\partial t}$ of $k[t, \theta]$ is homogeneous of degree -1 , and $\operatorname{Spec}\left(R\left(k[t], \frac{\partial}{\partial t}\right)\right)$ endowed with the corresponding $\mathbb{G}_{a}$-action is just the trivial $\mathbb{G}_{a}$-torsor over $\operatorname{Spec}(k[\theta])$ via the projection

$$
\pi=\operatorname{pr}_{\theta}: \operatorname{Spec}\left(R\left(k[t], \frac{\partial}{\partial t}\right)\right) \rightarrow \operatorname{Spec}(k[\theta]) .
$$

On the other hand, the locally nilpotent $k[\theta]$-derivation $\theta \frac{\partial}{\partial t}$ of $k[t, \theta]$ is homogeneous of degree 0 and the open immersion

$$
\mathbb{G}_{a}=\operatorname{Spec}(k[t]) \hookrightarrow \mathbb{P}^{1}=\operatorname{Proj}_{k}(k[t, \theta]), \quad t \mapsto[t: 1]
$$

is equivariant for the $\mathbb{G}_{a}$-action $t^{\prime} \cdot[t: \theta] \mapsto\left[t+t^{\prime} \theta: \theta\right]$ on $\mathbb{P}^{1}$ induced by $\theta \frac{\partial}{\partial t}$.

The content of the article is the following. In the first section we establish basic general properties of Rees algebras of additive group scheme actions in a relative and characteristic free setting. Namely, given a fixed base scheme or algebraic space $S$, we consider schemes or algebraic spaces $X$ that are endowed with an action of the additive group scheme $\mathbb{G}_{a}$ and which admit a $\mathbb{G}_{a}$-invariant affine morphism $f: X \rightarrow S$. Having this flexibility is useful even in the absolute case of an affine variety $X$ over a base field endowed with $\mathbb{G}_{a}$-action since we can then analyze the relative structure of $X$ with respect to any $\mathbb{G}_{a}$-invariant morphism $f: X \rightarrow S$ to some scheme or algebraic space. The second section focuses on the case of algebraic varieties over a field of characteristic zero. We study the behavior of Rees algebras under certain type of equivariant morphisms and characterize geometrically those that are finitely generated. We also describe an algorithm to compute generators of these algebras. The last section is devoted to a selection of examples which illustrate the interplay between relative and absolute Rees algebras. We also present an application of Rees algebras to the construction and classification of affine extensions of $\mathbb{G}_{a}$-torsors over punctured surfaces [11, 18], a 
class of varieties which form one of the building blocks of the classification theory of affine threefolds with $\mathbb{G}_{a}$-actions.

\section{CONTENTS}

Introduction 1

1. Rees algebras of affine $\mathbb{G}_{a}$-schemes 3

1.1. Additive group scheme actions on relative affine schemes 3

1.2. Rees algebras and associated graded homomorphisms 5

1.3. Associated canonical additive group actions $\quad 6$

1.4. Behavior with respect to equivariant morphisms $\quad 7$

1.5. Rees algebras of $\mathbb{G}_{a}$-torsors $\quad 8$

2. Rees algebras of affine $\mathbb{G}_{a}$-varieties over a field of characteristic zero 9

2.1. Basic properties of global Rees algebras of affine $\mathbb{G}_{a}$-varieties 9

2.2. Rees algebras and equivariant birational morphisms $\quad 11$

2.3. Finitely generated Rees algebras 12

2.4. Relation between global Rees algebras and relative Rees algebras of the fixed point free locus 16

3. Examples and Applications $r$

3.1. Danielewski hypersurfaces in $\mathbb{A}_{k}^{3} \quad 18$

3.2. A smooth affine threefold whose geometric quotient is quasi-projective but not quasi-affine 19

3.3. A triangular $\mathbb{G}_{a}$-action on $\mathbb{A}_{k}^{3}$ and the Russell cubic threefold 20

$\begin{array}{ll}\text { 3.4. Winkelmann's proper locally trivial action on } \mathbb{A}_{k}^{5} & 21\end{array}$

3.5. Extensions of $\mathbb{G}_{a}$-torsors over punctured surfaces 23

$\begin{array}{lr}\text { References } & 25\end{array}$

\section{ReES Algebras of AFFine $\mathbb{G}_{a}$-SCHEMES}

Given a scheme or an algebraic space $S$, we denote by $\mathbb{G}_{a, S}=S \times_{\mathbb{Z}} \mathbb{G}_{a, \mathbb{Z}}=\operatorname{Spec}\left(\mathcal{O}_{S}[t]\right)$ the additive group scheme over $S$. We denote by $m: \mathbb{G}_{a, S} \times{ }_{S} \mathbb{G}_{a, S} \rightarrow \mathbb{G}_{a, S}$ and $e: S \rightarrow \mathbb{G}_{a, S}$ its group law and neutral section respectively. By an affine $S$-scheme $f: X \rightarrow S$, we mean the relative spectrum of a quasi-coherent sheaf $\mathcal{A}=f_{*} \mathcal{O}_{X}$ of $\mathcal{O}_{S}$-algebras. We say that $X$ is of finite type over $S$ if $\mathcal{A}$ is locally of finite type as an $\mathcal{O}_{S}$-algebra.

1.1. Additive group scheme actions on relative affine schemes. Let $S$ be a scheme or an algebraic space. An action $\mu: \mathbb{G}_{a, S} \times{ }_{S} X \rightarrow X$ of $\mathbb{G}_{a, S}$ on an affine $S$-scheme $f: X=\operatorname{Spec}_{S}(\mathcal{A}) \rightarrow S$ is equivalently determined by its $\mathcal{O}_{S}$-algebra co-morphism

$$
\mu^{*}: \mathcal{A} \rightarrow \mathcal{A} \otimes \mathcal{O}_{S} \mathcal{O}_{S}[t]=\mathcal{A}[t]
$$

which satisfies the usual axioms of a group co-action, namely the commutativity of the following two diagrams:
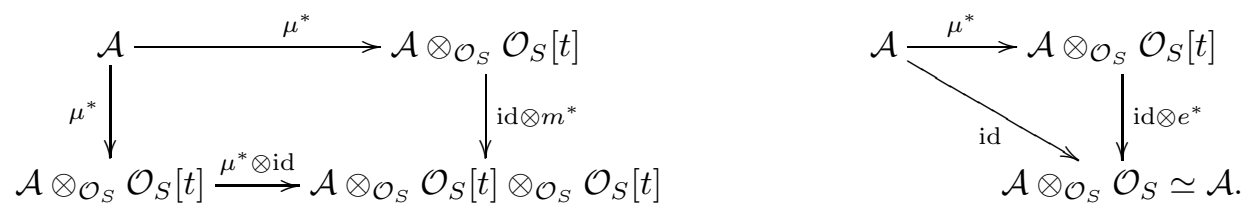

For every $i \geq 0$, let $\mathrm{p}_{i}: \mathcal{O}_{S}[t]=\bigoplus_{j=0}^{\infty} \mathcal{O}_{S} \rightarrow \mathcal{O}_{S}$ be the projection onto the $i$-th factor, and let $D^{(i)}=\left(\operatorname{id}_{\mathcal{A}} \otimes \mathrm{p}_{i}\right) \circ \mu^{*}: \mathcal{A} \rightarrow \mathcal{A}$. The following lemma is a well-known consequence of the commutativity of the above diagrams.

Lemma 1. The $\mathcal{O}_{S}$-module endomorphisms $D^{(i)}=\left(\operatorname{id}_{\mathcal{A}} \otimes \mathrm{p}_{i}\right) \circ \mu^{*}: \mathcal{A} \rightarrow \mathcal{A}$ are differential operators of order $\leq i$ which satisfy the following properties: 
(1) The operator $D^{(0)}$ is the identity map of $\mathcal{A}$,

(2) For every $i \geq 0$, the Leibniz rule $D^{(i)}(a b)=\sum_{j=0}^{i} D^{(j)}(a) D^{(i-j)}(b)$ holds for every pair of local sections $a, b$ of $\mathcal{A}$ over $S$,

(3) For every $i, j \geq 0, D^{(i)} \circ D^{(j)}=\left(\begin{array}{c}i+j \\ i\end{array}\right) D^{(i+j)}$,

(4) We have $\mathcal{A}=\bigcup_{n \geq 0}\left(\bigcap_{i>n} \operatorname{Ker} D^{(i)}\right)$.

Proof. The fact that $D^{(0)}=\mathrm{id}_{\mathcal{A}}$ follows from the commutativity of the diagram on the right in (1.1). Given local sections $a, b$ of $\mathcal{A}$ over $S$, the fact that $\mu^{*}$ is a $\mathcal{O}_{S}$-algebra homomorphism implies that $\mu^{*}(a b)=$ $\mu^{*}(a) \mu^{*}(b)$. Writing $\mu^{*}(a)=\sum_{i \geq 0} D^{(i)}(a) t^{i}, \mu^{*}(b)=\sum_{i \geq 0} D^{(i)}(b) t^{i}$ and $\mu^{*}(a b)=\sum_{i \geq 0} D^{(i)}(a b) t^{i}$, we have

$$
D^{(i)}(a b)=\sum_{j=0}^{i} D^{(j)}(a) D^{(i-j)}(b)
$$

for every $i \geq 0$, which proves Property (2). Let $a$ be a local section of $\mathcal{A}$ over $S$ and write $\mu^{*}(a)=$ $\sum_{i \geq 0} D^{(i)}(a) t^{i}$ and for every $i \geq 0, \mu^{*}\left(D^{(i)}(a)\right)=\sum_{j \geq 0} D^{(j)}\left(D^{(i)}(a)\right) v^{j}$. The commutativity of the diagram on the left in (1.1) implies that

$$
\sum_{i \geq 0} D^{(i)}(a)(t+v)^{i}=\sum_{i \geq 0}\left(\sum_{j \geq 0} D^{(j)}\left(D^{(i)}(a)\right) v^{j}\right) t^{i}
$$

from which it follows, by identifying the terms in $t^{i} v^{j}$, that $D^{(j)}\left(D^{(i)}(a)\right)=\left(\begin{array}{c}i+j \\ i\end{array}\right) D^{(i+j)}(a)$. This proves Property (3). For every $n \geq 0, \mathcal{F}_{n}=\bigcap_{i>n} \mathcal{K} \operatorname{er} D^{(i)}$ is an $\mathcal{O}_{S}$-submodule of $\mathcal{A}$ which is equal to the inverse image by $\mu^{*}$ of the $\mathcal{O}_{S}$-submodule $\mathcal{A} \otimes \mathcal{O}_{S} \mathcal{O}_{S}[t]_{\leq n}$ of $\mathcal{A} \otimes \mathcal{O}_{S} \mathcal{O}_{S}[t]$. The union $\bigcup_{n \geq 0} \mathcal{F}_{n}$ is an $\mathcal{O}_{S}$-submodule of $\mathcal{A}$ which is stable under multiplication by Property (2), hence an $\mathcal{O}_{S}$-subalgebra of $\mathcal{A}$. The fact that the inclusion $\bigcup_{n \geq 0} \mathcal{F}_{n} \hookrightarrow \mathcal{A}$ is an isomorphism of $\mathcal{O}_{S}$-algebras is then checked on an open cover of $S$ by affine open subsets as in [26]. This proves Property (4).

Definition 2. A collection of $\mathcal{O}_{S}$-linear differential operators $D^{(i)}: \mathcal{A} \rightarrow \mathcal{A}, i \in \mathbb{Z}_{\geq 0}$ which satisfy the properties of Lemma 1 is called a locally finite iterative higher $\mathcal{O}_{S}$-derivation $\left(\mathcal{O}_{S}\right.$-LFIHD for short) of the quasi-coherent $\mathcal{O}_{S}$-algebra $\mathcal{A}$.

Conversely, for every $\mathcal{O}_{S}$-LFIHD $D=\left\{D^{(i)}\right\}_{i \geq 0}$ of a quasi-coherent $\mathcal{O}_{S}$-algebra $\mathcal{A}$, the exponential map

$$
\mu^{*}:=\exp (t D)=\sum_{i=0}^{\infty} D^{(i)} \otimes t^{i}: \mathcal{A} \rightarrow \mathcal{A} \otimes \mathcal{O}_{S} \mathcal{O}_{S}[t], \quad a \mapsto \sum_{i=0}^{\infty} D^{(i)}(a) \otimes t^{i}
$$

is the co-morphism of a $\mathbb{G}_{a, S^{-}}$-action $\mu: \mathbb{G}_{a, S} \times_{S} X \rightarrow X$ on $X$ (see e.g. [26]).

The quasi-coherent $\mathcal{O}_{S}$-algebra $\mathcal{A}$ of an affine $S$-scheme $f: X=\operatorname{Spec}_{S}(\mathcal{A}) \rightarrow S$ equipped with a $\mathbb{G}_{a, S}$-action is endowed with an increasing exhaustive filtration by its $\mathcal{O}_{S}$-submodules

$$
\mathcal{F}_{n}=\left(\mu^{*}\right)^{-1}\left(\mathcal{A} \otimes \mathcal{O}_{S} \mathcal{O}_{S}[t]_{\leq n}\right)=\bigcap_{i>n} \mathcal{K} \operatorname{er} D^{(i)}, \quad n \in \mathbb{Z}_{\geq 0},
$$

consisting of elements whose image by $\mu^{*}$ are polynomials with coefficients in $\mathcal{A}$ of degree less than or equal to $n$ in the variable $t$. The following lemma, whose proof is left to the reader, records some basic properties of this filtration.

Lemma 3. With the above notation, the following hold:

a) For every $m, n \geq 0$, we have $\mathcal{F}_{m} \cdot \mathcal{F}_{n} \subseteq \mathcal{F}_{m+n}$, where $\cdot$ denotes the product law in $\mathcal{A}$,

b) For every $i \geq 0$ and $n \geq 0$, we have $D^{(i)} \mathcal{F}_{n} \subseteq \mathcal{F}_{n-i}$,

c) The submodule $\mathcal{F}_{0}$ is an $\mathcal{O}_{S}$-subalgebra of $\overline{\mathcal{A}}$ which coincides with the $\mathcal{O}_{S}$-algebra $\mathcal{A}_{0}=\mathcal{A}^{\mathbb{G}_{a, S}}$ of germs of $\mathbb{G}_{a, S}$-invariant morphisms $X \rightarrow \mathbb{A}_{S}^{1}$,

d) Each $\mathcal{F}_{n}$ is naturally endowed with an additional structure of $\mathcal{A}_{0}$-module,

e) For every $n \geq 0$, the image of $\left.\mu^{*}\right|_{\mathcal{F}_{n}}: \mathcal{F}_{n} \rightarrow \mathcal{A} \otimes \mathcal{O}_{S}[t]_{\leq n}$ is contained in $\mathcal{F}_{n} \otimes \mathcal{O}_{S} \mathcal{O}_{S}[t]_{\leq n}$. Moreover $\left.\mu^{*}\right|_{\mathcal{A}_{0}}: \mathcal{A}_{0} \rightarrow \mathcal{A}_{0} \otimes_{\mathcal{O}_{S}} \mathcal{O}_{S}[t]_{\leq 0} \cong \mathcal{A}_{0}$ is an isomorphism onto its image. 


\subsection{Rees algebras and associated graded homomorphisms.}

Definition 4. Let $f: X=\operatorname{Spec}_{S}(\mathcal{A}) \rightarrow S$ be an affine $S$-scheme endowed with a $\mathbb{G}_{a, S}$-action $\mu: \mathbb{G}_{a, S} \times_{S}$ $X \rightarrow X$, let $D=\left\{D^{(i)}\right\}_{i \geq 0}$ and let $\left\{\mathcal{F}_{n}=\bigcap_{i>n} \mathcal{K} \text { er } D^{(i)}\right\}_{n \geq 0}$ be the corresponding $\mathcal{O}_{S}$-LFIHD and filtration of $\mathcal{A}$ respectively.

1) The Rees algebra of $(X, \mu)$ is the sheaf of graded $\mathcal{A}_{0}$-algebras $\mathcal{R}(X, \mu)=\bigoplus_{n=0}^{\infty} \mathcal{F}_{n}$, equipped with the multiplication induced by that of $\mathcal{A}$.

2) The associated graded algebra of $(X, \mu)$ is the sheaf of graded $\mathcal{A}_{0}$-algebras $\mathcal{G} r(X, \mu)=\bigoplus_{n=0}^{\infty} \mathcal{F}_{n} / \mathcal{F}_{n-1}$, where by convention $\mathcal{F}_{-1}=\{0\}$, equipped with the multiplication induced by that of $\mathcal{A}$.

The collections of inclusions $\gamma_{n}: \mathcal{A}_{0}=\mathcal{F}_{0} \hookrightarrow \mathcal{F}_{n}$ and $\eta_{n}: \mathcal{F}_{n} \hookrightarrow \mathcal{A}, n \geq 0$ induce respective injective graded $\mathcal{A}_{0}$-algebra homomorphisms

$$
\mathcal{A}_{0}[\theta]=\bigoplus_{n=0}^{\infty} \mathcal{A}_{0} \stackrel{\gamma}{\longrightarrow} \mathcal{R}(X, \mu) \stackrel{\eta}{\longrightarrow} \bigoplus_{n=0}^{\infty} \mathcal{A} \stackrel{\cong}{\rightrightarrows} \mathcal{A}[\theta]
$$

of degree 0 , where $\mathcal{A}[\theta] \rightarrow \bigoplus_{n=0}^{\infty} \mathcal{A}$ is the isomorphism of graded $\mathcal{A}$-algebras which maps the variable $\theta$ to the constant section $1 \in \Gamma\left(S, \mathcal{O}_{S}\right) \subset \Gamma\left(S, \mathcal{A}_{0}\right) \subset \Gamma(S, \mathcal{A})$ viewed in degree 1 . This provides an identification of $\mathcal{R}(X, \mu)$ with the $\mathcal{A}_{0}[\theta]$-subalgebra

$$
\bigoplus_{n=0}^{\infty} \mathcal{F}_{n} \theta^{n} \subset \mathcal{A}[\theta]
$$

consisting of polynomials $p=\sum a_{n} \theta^{n}$ in the variable $\theta$ such that $a_{n} \in \mathcal{F}_{n} \subset \mathcal{A}$ for every $n \geq 0$.

Lemma 5. With the above notation, the following hold:

1) The kernel of the surjective graded $\mathcal{A}_{0}$-algebra homomorphism $q_{0}: \mathcal{R}(X, \mu) \rightarrow \mathcal{G} r(X, \mu)$ of degree 0 induced by the collection of quotient homomorphisms $\mathcal{F}_{n} \rightarrow \mathcal{F}_{n} / \mathcal{F}_{n-1}, n \geq 0$, is equal to the homogeneous ideal sheaf $\theta \mathcal{R}(X, \mu)$ of $\mathcal{R}(X, \mu)$.

2) The quotient of $\mathcal{R}(X, \mu)$ by the ideal sheaf $(1-\theta) \mathcal{R}(X, \mu)$ is canonically isomorphic to $\mathcal{A}$, and the restriction of quotient homomorphism $q_{1}: \mathcal{R}(X, \mu) \rightarrow \mathcal{A}$ to each homogeneous piece $\mathcal{F}_{n}$ is an isomorphism onto its image in $\mathcal{A}$.

Proof. By definition, we have

$$
\mathcal{R}(X, \mu) / \theta \mathcal{R}(X, \mu) \cong \bigoplus_{n \geq 0}\left(\mathcal{F}_{n} \theta^{n}\right) /\left(\mathcal{F}_{n-1} \theta^{n}\right) \cong \bigoplus_{n \geq 0}\left(\mathcal{F}_{n} / \mathcal{F}_{n-1}\right) \theta^{n} \cong \mathcal{G} r(X, \mu) .
$$

The injective homomorphism $\eta: \mathcal{R}(X, \mu) \rightarrow \mathcal{A}[\theta]$ induces an injective homomorphism

$$
\eta_{(\theta)}: \mathcal{R}(X, \mu)_{(\theta)} \rightarrow \mathcal{A}[\theta]_{(\theta)}
$$

between the degree 0 parts of the localizations of $\mathcal{R}(X, \mu)$ and $\mathcal{A}[\theta]$ respectively with respect to the homogeneous element $\theta \in \mathcal{F}_{1}$. By [16, Proposition 2.2.5], we have canonical isomorphisms

$$
\mathcal{R}(X, \mu) /(1-\theta) \mathcal{R}(X, \mu) \rightarrow \mathcal{R}(X, \mu)_{(\theta)}, \quad \overline{\mathcal{F}}_{n} \ni \bar{f}_{n} \mapsto f_{n} / \theta^{n}
$$

and

$$
\mathcal{A}[\theta] /(1-\theta) \mathcal{A}[\theta] \rightarrow \mathcal{A}[\theta]_{(\theta)} \cong \mathcal{A}, \quad \overline{a_{n} \theta^{n}} \mapsto a_{n} .
$$

Via these canonical isomorphisms, the homomorphism $q_{1}: \mathcal{R}(X, \mu) \rightarrow \mathcal{A}$ coincides with the composition of the localization homomorphism

$$
\mathcal{R}(X, \mu) \rightarrow \mathcal{R}(X, \mu)_{(\theta)}, \quad \mathcal{F}_{n} \ni f_{n} \mapsto f_{n} / \theta^{n}
$$

with $\eta_{(\theta)}: \mathcal{R}(X, \mu)_{(\theta)} \rightarrow \mathcal{A}[\theta]_{(\theta)}$. The second assertion then follows since $\mathcal{A}=\bigcup_{n \geq 0} \mathcal{F}_{n}$ by hypothesis. 
Let $f_{0}: X_{0}=\operatorname{Spec}_{S}\left(\mathcal{A}_{0}\right) \rightarrow S$ be the relative spectrum of the $\mathcal{O}_{S}$-subalgebra $\mathcal{A}_{0}$ of $\mathcal{A}$. The closed immersions $i_{1}: X \hookrightarrow \operatorname{Spec}_{S}(\mathcal{R}(X, \mu))$ and $i_{0}: \operatorname{Spec}_{S}(\mathcal{G} r(X, \mu)) \hookrightarrow \operatorname{Spec}_{S}(\mathcal{R}(X, \mu))$ defined by $q_{1}$ and $q_{0}$ respectively fit in a commutative diagram

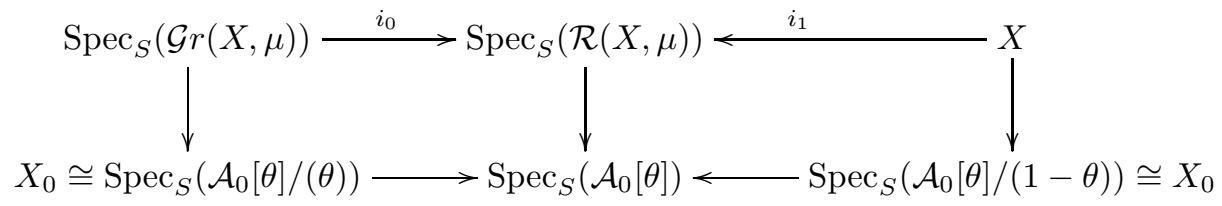

whose left-hand and right-hand squares are cartesian.

Lemma 6. The graded homomorphisms $q_{0}: \mathcal{R}(X, \mu) \rightarrow \mathcal{G} r(X, \mu)$ and $\eta: \mathcal{R}(X, \mu) \rightarrow \mathcal{A}[\theta]$ induce respectively:

1) A closed immersion $\bar{i}_{0}: \operatorname{Proj}_{S}(\mathcal{G r}(X, \mu)) \rightarrow \operatorname{Proj}_{S}(\mathcal{R}(X, \mu))$ with image equal to Weil divisor $V_{+}(\theta)$,

2) An open embedding $j: X \cong \operatorname{Proj}_{S}(\mathcal{A}[\theta]) \hookrightarrow \operatorname{Proj}_{S}(\mathcal{R}(X, \mu))$ with image equal to the open subset $D_{+}(\theta)=\operatorname{Proj}_{S}(\mathcal{R}(X, \mu)) \backslash V_{+}(\theta)$.

Proof. The first assertion is clear. For the second, we observe that

$$
\operatorname{Proj}(\eta): \operatorname{Proj}_{S}(\mathcal{A}[\theta]) \rightarrow \operatorname{Proj}_{S}(\mathcal{R}(X, \mu))
$$

is the composition of the canonical isomorphisms

$$
\operatorname{Proj}_{S}(\mathcal{A}[\theta]) \cong \operatorname{Spec}_{S}(\mathcal{A}) \cong \operatorname{Spec}_{S}\left(\mathcal{A}[\theta]_{(\theta)}\right) \stackrel{\operatorname{Spec}\left(\eta_{(\theta)}\right)}{\longrightarrow} \operatorname{Spec}_{S}\left(\mathcal{R}(X, \mu)_{(\theta)}\right)=D_{+}(\theta)
$$

with the embedding $D_{+}(\theta) \hookrightarrow \operatorname{Proj}_{S}(\mathcal{R}(X, \mu))$.

1.3. Associated canonical additive group actions. Let $f: X=\operatorname{Spec}_{S}(\mathcal{A}) \rightarrow S$ be an affine $S$-scheme endowed with a $\mathbb{G}_{a, S}$-action $\mu: \mathbb{G}_{a, S} \times_{S} X \rightarrow X$. Let $D=\left\{D^{(i)}\right\}_{i>0}$ be the corresponding $\mathcal{O}_{S}$-LFIHD of $\mathcal{A}$ and $\mathcal{R}(X, \mu)=\bigoplus_{n=0}^{\infty} \mathcal{F}_{n}$ be the Rees algebra of $(X, \mu)$.

Since $D^{(i)} \mathcal{F}_{n} \subseteq \mathcal{F}_{n-i}$ by Lemma (3) b), the $\mathcal{O}_{S}$-LFIHD $D$ induces an homogeneous $\mathcal{O}_{S}$-LFIHD

$$
\mathcal{R}(D): \mathcal{R}(X, \mu) \rightarrow \mathcal{R}(X, \mu)
$$

of degree -1 with respect to the grading of $\mathcal{R}(X, \mu)$, defined by $\left.\mathcal{R}(D)^{(i)}\right|_{\mathcal{F}_{n}}=\left.D^{(i)}\right|_{\mathcal{F}_{n}}$ for every $i, n \geq 0$. Furthermore, $\mathcal{R}(D)$ induces via the quotient morphism $q_{0}: \mathcal{R}(X, \mu) \rightarrow \mathcal{G} r(X, \mu)$ an $\mathcal{O}_{S}$-LFIHD $\operatorname{gr}(D)$ of $\mathcal{G} r(X, \mu)$ which is also homogeneous of degree -1 . By construction, we have the following:

Lemma 7. Let $f: X=\operatorname{Spec}_{S}(\mathcal{A}) \rightarrow S$ be an affine $S$-scheme endowed with a $\mathbb{G}_{a, S}$-action $\mu: \mathbb{G}_{a, S} \times_{S}$ $X \rightarrow X$ with associated $\mathcal{O}_{S}-L F I H D D$ of $\mathcal{A}$. Then the closed immersions

$$
X \stackrel{i_{1}}{\hookrightarrow} \operatorname{Spec}_{S}(\mathcal{R}(X, \mu)) \stackrel{i_{0}}{\hookleftarrow} \operatorname{Spec}_{S}(\mathcal{G r}(X, \mu))
$$

are equivariant for the $\mathbb{G}_{a, S}$-actions on $X, \operatorname{Spec}_{S}(\mathcal{R}(X, \mu))$ and $\operatorname{Spec}_{S}(\mathcal{G} r(X, \mu))$ associated respectively to the $\mathcal{O}_{S}-L F I H D D, \mathcal{R}(D)$ and $\operatorname{gr}(D)$.

For every $i \geq 0$, let $\theta^{i} \mathcal{R}(D)^{(i)}$ denote the $\mathcal{O}_{S}$-linear differential operator of $\mathcal{R}(X, \mu)$ whose restriction to $\mathcal{F}_{n}$ is equal to the composition of $\mathcal{R}(D)^{(i)}: \mathcal{F}_{n} \rightarrow \mathcal{F}_{n-i}$ with the natural inclusion $\mathcal{F}_{n-i} \hookrightarrow \mathcal{F}_{n}$. The collection $\theta \mathcal{R}(D)=\left\{\theta^{i} \mathcal{R}(D)^{(i)}\right\}_{i>0}$ is then an $\mathcal{O}_{S}$-LFIHD of $\mathcal{R}(X, \mu)$ which is homogeneous of degree 0 with respect to the grading. Note that it induces the trivial $\mathcal{O}_{S}$-LFIHD on $\mathcal{G} r(X, \mu)$. Since $\theta \mathcal{R}(D)$ is homogeneous of degree 0 , it defines a $\mathbb{G}_{a, S^{-}}$action on $\operatorname{Spec}_{S}(\mathcal{R}(X, \mu))$ which commutes with the $\mathbb{G}_{m, S^{-}}$ action associated to the grading of $\mathcal{R}(X, \mu)$. The $\mathbb{G}_{a, S^{-}}$action on $\operatorname{Spec}_{S}(\mathcal{R}(X, \mu))$ thus descends to a $\mathbb{G}_{a, S^{-}}$ action on $\operatorname{Proj}_{S}(\mathcal{R}(X, \mu))$.

Lemma 8. Let $f: X=\operatorname{Spec}_{S}(\mathcal{A}) \rightarrow S$ be an affine $S$-scheme endowed with a $\mathbb{G}_{a, S}$-action $\mu: \mathbb{G}_{a, S} \times_{S}$ $X \rightarrow X$ with associated $\mathcal{O}_{S}-L F I H D D$ of $\mathcal{A}$. Then the open embedding $j: X \hookrightarrow \operatorname{Proj}_{S}(\mathcal{R}(X, \mu))$ of Lemma 6 is equivariant for the $\mathbb{G}_{a, S}$-actions on $X$ and $\operatorname{Proj}_{S}(\mathcal{R}(X, \mu))$ determined respectively by $D$ and the homogeneous $\mathcal{O}_{S}$-LFIHD $\theta \mathcal{R}(D)$. 
Proof. Viewing $\mathcal{R}(X, \mu)$ as a subalgebra of $\mathcal{A}[\theta]$ via the injective homomorphism $\eta: \mathcal{R}(X, \mu) \rightarrow \mathcal{A}[\theta]$ in (1.2), the $\mathcal{O}_{S}$-LFIHD $\theta \mathcal{R}(D)$ coincides with restriction to $\mathcal{R}(X, \mu)$ of the $\mathcal{O}_{S}$-LFIHD $D \otimes$ id of $\mathcal{A}[\theta]=$ $\mathcal{A} \otimes \mathcal{O}_{S} \mathcal{O}_{S}[\theta]$ corresponding to the $\mathbb{G}_{a, S}$-action on $X \times_{S} \mathbb{A}_{S}^{1}=\operatorname{Spec}_{S}(\mathcal{A}[\theta])$ defined as the product of the $\mathbb{G}_{a, S}$-action $\mu$ on $X$ with the trivial $\mathbb{G}_{a, S}$-action on the second factor. The open embedding $j=\operatorname{Proj}(\eta)$ : $\operatorname{Proj}_{S}(\mathcal{A}[\theta]) \rightarrow \operatorname{Proj}_{S}(\mathcal{R}(X, \mu))$ of Lemma 6 is thus equivariant for the corresponding $\mathbb{G}_{a, S}$-actions. The assertion follows since the canonical isomorphism $X \cong \operatorname{Proj}_{S}(\mathcal{A}[\theta])$ is equivariant for the $\mathbb{G}_{a, S}$-actions determined by $D$ and $D \otimes$ id respectively.

1.4. Behavior with respect to equivariant morphisms. Let $h: S^{\prime} \rightarrow S$ be a morphism of schemes or algebraic spaces and let $\tilde{h}: \mathbb{G}_{a, S^{\prime}} \rightarrow \mathbb{G}_{a, S}$ be the homomorphism of group schemes it induces. Let $f$ : $X \rightarrow S$ (resp. $f^{\prime}: X^{\prime} \rightarrow S^{\prime}$ ) be an affine $S$-scheme (resp. affine $S^{\prime}$-scheme) and assume that $X$ and $X^{\prime}$ are endowed with actions $\mu$ and $\mu^{\prime}$ of $\mathbb{G}_{a, S}$ and $\mathbb{G}_{a, S^{\prime}}$ respectively.

Definition 9. With the above notation, a morphism $g: X^{\prime} \rightarrow X$ such that $h \circ f^{\prime}=f \circ g$ is called $\mathbb{G}_{a^{-}}$ equivariant if the following diagram commutes

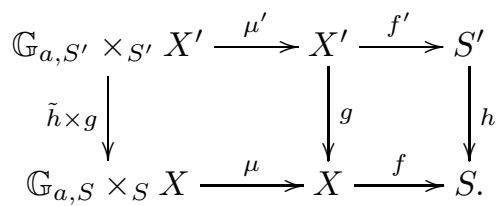

Letting $\mathcal{A}=f_{*} \mathcal{O}_{X}$ and $\mathcal{A}^{\prime}=f_{*}^{\prime} \mathcal{O}_{X^{\prime}}$, a morphism $g: X^{\prime} \rightarrow X$ such that $h \circ f^{\prime}=f \circ g$ is uniquely determined by its $\mathcal{O}_{S}$-algebra co-morphism $g^{*}: \mathcal{A} \rightarrow h_{*} \mathcal{A}^{\prime}$. Let $D=\left\{D^{(i)}\right\}_{i \geq 0}$ and $D^{\prime}=\left\{D^{\prime(i)}\right\}_{i \geq 0}$ be the $\mathcal{O}_{S}$-LFIHD and $\mathcal{O}_{S^{\prime}}$-LFIHD determining the actions $\mu$ and $\mu^{\prime}$, and let $\left\{\mathcal{F}_{n}\right\}_{n \geq 0}$ and $\left\{\mathcal{F}_{n}^{\prime}\right\}_{\geq 0}$ be the associated ascending filtrations of $\mathcal{A}$ and $\mathcal{A}^{\prime}$ respectively.

Lemma 10. A morphism $g: X^{\prime} \rightarrow X$ such that $h \circ f^{\prime}=f \circ g$ is $\mathbb{G}_{a}$-equivariant if and only if it satisfies the following equivalent conditions:

1) For every $i \geq 0, h_{*} D^{\prime(i)} \circ g^{*}=g^{*} \circ D^{(i)}$,

2) For every $n \geq 0, g^{*} \mathcal{F}_{n} \subseteq h_{*} \mathcal{F}_{n}^{\prime}$.

Proof. By definition, the morphism $\tilde{h}: \mathbb{G}_{a, S^{\prime}} \rightarrow \mathbb{G}_{a, S}$ is determined by the homomorphism

$$
h^{*} \otimes \mathrm{id}: \mathcal{O}_{S}[t]=\mathcal{O}_{S} \otimes_{\mathbb{Z}} \mathbb{Z}[t] \rightarrow\left(h_{*} \mathcal{O}_{S^{\prime}}\right) \otimes_{\mathbb{Z}} \mathbb{Z}[t]=\tilde{h}_{*}\left(\mathcal{O}_{S^{\prime}}[t]\right),
$$

where $h^{*}: \mathcal{O}_{S} \rightarrow h_{*} \mathcal{O}_{S^{\prime}}$ is the $\mathcal{O}_{S}$-module homomorphism in the definition of $h$. The commutativity of the diagram (1.3) is then equivalent to that of the following diagram of $\mathcal{O}_{S}$-algebra homomorphisms

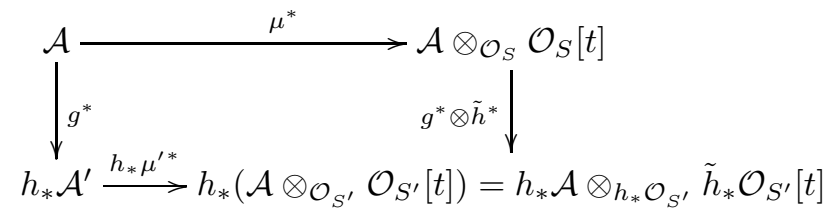

from which the claimed equivalences follow.

By Lemma 10, the comorphism $g^{*}: \mathcal{A} \rightarrow h_{*} \mathcal{A}^{\prime}$ of a $\mathbb{G}_{a}$-equivariant morphism $g: X^{\prime} \rightarrow X$ is thus a homomorphism of filtered $\mathcal{O}_{S}$-algebras of degree 0 with respect to the filtrations $\left\{\mathcal{F}_{n}\right\}_{n \geq 0}$ and $\left\{h_{*} \mathcal{F}_{n}^{\prime}\right\}_{n \geq 0}$ associated to the actions $\mu$ and $\mu^{\prime}$ respectively. As a consequence, $g^{*}$ induces a homomorphism of $\mathcal{O}_{S^{-}}$ algebras $g_{0}^{*}: \mathcal{A}_{0} \rightarrow h_{*} \mathcal{A}_{0}^{\prime}$ and homomorphisms of graded algebras

$$
\mathcal{R}(g): \mathcal{R}(X, \mu) \rightarrow h_{*} \mathcal{R}\left(X^{\prime}, \mu^{\prime}\right) \quad \text { and } \operatorname{gr}(g): \mathcal{G} r(X, \mu) \rightarrow h_{*} \mathcal{G} r\left(X^{\prime}, \mu^{\prime}\right),
$$


both of degree 0 . Furthermore, with the notation of (1.2), we have a commutative diagram

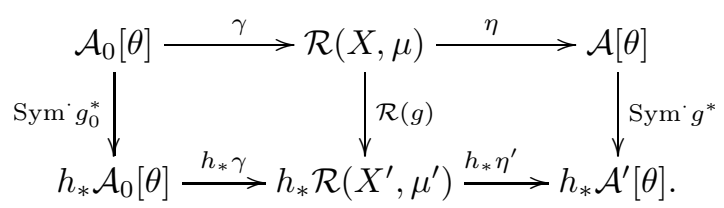

The following proposition is a direct consequence of the definitions given in subsection 1.3.

Proposition 11. Let $f: X=\operatorname{Spec}_{S}(\mathcal{A}) \rightarrow S$ and $f^{\prime}: X^{\prime}=\operatorname{Spec}_{S^{\prime}}\left(\mathcal{A}^{\prime}\right) \rightarrow S^{\prime}$ be affine schemes over $S$ and

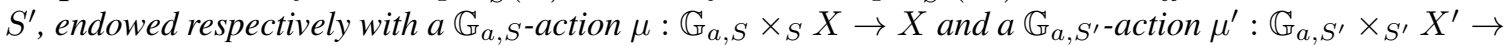

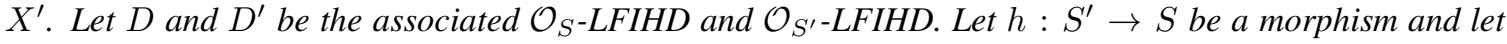
$g: X^{\prime} \rightarrow X$ be a $\mathbb{G}_{a}$-equivariant morphism. Then the following hold:

1) The diagram

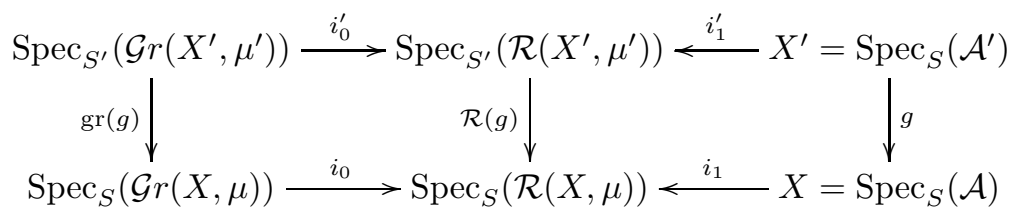

is commutative and equivariant for the $\mathbb{G}_{a}$-actions defined by the $\mathcal{O}_{S^{\prime}}-L F I H D \operatorname{gr} r\left(D^{\prime}\right), D^{\prime}$ and $\mathcal{R}\left(D^{\prime}\right)$ and the $\mathcal{O}_{S}-L F I H D \operatorname{gr}(D), D$ and $\mathcal{R}(D)$ respectively.

2) The diagram

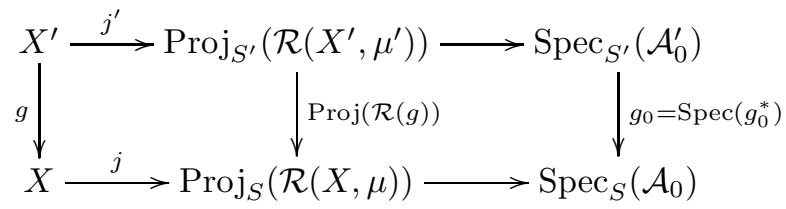

is commutative and equivariant for the $\mathbb{G}_{a}$-actions defined by the $\mathcal{O}_{S^{\prime}}-L F I H D D^{\prime}$ and $\theta \mathcal{R}\left(D^{\prime}\right)$ and the $\mathcal{O}_{S^{-}}$ LFIHD $D$ and $\theta \mathcal{R}(D)$ respectively.

1.5. Rees algebras of $\mathbb{G}_{a}$-torsors. Recall that a $\mathbb{G}_{a, S}$-torsor is an $S$-scheme $f: P \rightarrow S$ endowed with a $\mathbb{G}_{a, S}$-action $\mu: \mathbb{G}_{a, S} \times{ }_{S} P \rightarrow P$ which, étale locally over $S$, is equivariantly isomorphic to $\mathbb{G}_{a, S}$ acting on itself by translations. In particular, $P$ is an affine $S$-scheme of finite type. Let $\mathcal{A}=f_{*} \mathcal{O}_{P}$, and let $D$ and $\left\{\mathcal{F}_{n}\right\}_{n \geq 0}$ be the $\mathcal{O}_{S}$-LFIHD and the ascending filtration of $\mathcal{A}$ associated to the $\mathbb{G}_{a, S}$-action $\mu$. Since $P$ is étale locally isomorphic to $\mathbb{G}_{a, S}$ acting on itself by translations, we have $\mathcal{F}_{0}=\mathcal{A}_{0}=\mathcal{A}^{\mathbb{G}_{a, S}}=\mathcal{O}_{S}$.

Proposition 12. With the above notation, the following hold:

a) The $\mathcal{O}_{S}$-module $\mathcal{F}_{1}$ is an étale locally free sheaf of rank 2 and we have an exact sequence of $\mathcal{O}_{S}$-modules

$$
0 \rightarrow \mathcal{O}_{S}=\mathcal{F}_{0} \hookrightarrow \mathcal{F}_{1} \stackrel{D^{(1)}}{\rightarrow} \mathcal{F}_{0} \rightarrow 0 .
$$

b) The Rees algebra $\mathcal{R}(P, \mu)$ is canonically isomorphic to the symmetric algebra $\mathrm{Sym} \mathcal{F}_{1}=\bigoplus_{n=0}^{\infty} \mathrm{Sym}^{n} \mathcal{F}_{1}$ of $\mathcal{F}_{1}$.

c) The open immersion $j: P \hookrightarrow \operatorname{Proj}_{S}(\mathcal{R}(P, \mu))$ coincides with the open immersion of $P$ in the projective bundle $p: \mathbb{P}\left(\mathcal{F}_{1}\right)=\operatorname{Proj}_{S}\left(\operatorname{Sym}_{\mathcal{F}} \mathcal{F}_{1}\right) \rightarrow S$ as the complement of the section $S \rightarrow \mathbb{P}\left(\mathcal{F}_{1}\right)$ determined by the surjective homomorphism $D^{(1)}: \mathcal{F}_{1} \rightarrow \mathcal{O}_{S}$.

Proof. Since the surjectivity of the homomorphisms $D^{(1)}: \mathcal{F}_{1} \rightarrow \mathcal{F}_{0}$ and $\operatorname{Sym} \mathcal{F}_{1} \rightarrow \mathcal{R}(P, \mu)$ are local properties on $S$ with respect to the étale topology, to prove a) and b), it suffices to consider the case where $X \rightarrow S$ is the trivial $\mathbb{G}_{a, S}$-torsor $\operatorname{Spec}_{S}\left(\mathcal{O}_{S}[t]\right)$ with the $\mathbb{G}_{a, S}$-action given by the group structure $m$ : $\mathbb{G}_{a, S} \times_{S} \mathbb{G}_{a, S} \rightarrow \mathbb{G}_{a, S}$. Here the corresponding $\mathcal{O}_{S}$-LFIHD $D$ is given by the collection of differential operators

$$
D^{(i)}=\left.\frac{1}{i !} \frac{\partial^{i}}{\partial t^{i}}\right|_{t=0}, \quad i \geq 0
$$


which associate to a polynomial $p(t)$ the $i$-th term of its Taylor expansion at 0 . We thus have $\mathcal{F}_{n}=\mathcal{O}_{S}[t]_{\leq n}$, $n \geq 0$. In particular, $\mathcal{F}_{1}$ is the free $\mathcal{O}_{S}$-module of rank 2 generated by 1 and $t$, with $D^{(1)}(t)=1$, which proves a). We then have $\mathcal{F}_{n} \cong \operatorname{Sym}^{n} \mathcal{F}_{1}$ as $\mathcal{O}_{S}$-modules, and so $\mathcal{R}\left(\mathbb{G}_{a, S}, m\right)=\operatorname{Sym}^{\cdot} \mathcal{F}_{1} \cong \mathcal{O}_{S}[\theta, t]$ from which assertion b) follows. Note that the $\mathcal{O}_{S}$-LFIHD $\mathcal{R}(D)$ and $\theta \mathcal{R}(D)$ on Sym $\mathcal{F}_{1}$ are then given locally by $\mathcal{R}(D)^{(i)}=\left.\frac{1}{i !} \frac{\partial^{i}}{\partial t^{i}}\right|_{t=0}$ and $(\theta \mathcal{R}(D))^{(i)}=\left.\theta^{i} \frac{1}{i !} \frac{\partial^{i}}{\partial t^{i}}\right|_{t=0}$ respectively.

The section of $\mathbb{P}\left(\mathcal{F}_{1}\right)$ defined by the surjective homomorphism of $\mathcal{O}_{S}$-modules $D^{(1)}: \mathcal{F}_{1} \rightarrow \mathcal{O}_{S}$ is given by the closed immersion

$$
S \cong \operatorname{Proj}_{S}\left(\mathcal{O}_{S}[t]\right) \rightarrow \mathbb{P}\left(\mathcal{F}_{1}\right)=\operatorname{Proj}_{S}\left(\operatorname{Sym}_{\mathcal{F}} \mathcal{F}_{1}\right)
$$

determined by the surjective homomorphism of graded $\mathcal{O}_{S}$-algebras $\operatorname{Sym} \cdot\left(D^{(1)}\right): \operatorname{Sym} \mathcal{F}_{1} \rightarrow \operatorname{Sym} \cdot \mathcal{O}_{S} \cong$ $\mathcal{O}_{S}[t]$. By the previous description, the homomorphism $\operatorname{Sym} \cdot\left(D^{(1)}\right)$ coincides locally over $S$ with the homomorphism $\mathcal{O}_{S}[\theta, t] \rightarrow \mathcal{O}_{S}[t]$ with kernel $\theta \mathcal{O}_{S}[\theta, t]$. It follows that the kernel of $\operatorname{Sym}^{\cdot}\left(D^{(1)}\right)$ coincides via the isomorphism $\mathcal{R}(P, \mu) \cong \mathrm{Sym}^{\cdot} \mathcal{F}_{1}$ with the homogeneous ideal sheaf $\theta \mathcal{R}(P, \mu)$ of $\mathcal{R}(P, \mu)$, with quotient $\mathcal{R}(P, \mu) / \theta \mathcal{R}(P, \mu) \cong \mathcal{G} r(P, \mu)$. Assertion $\mathrm{c})$ is then a consequence of Lemma 6 .

Remark 13. It follows from Proposition 12 a) and c) that the isomorphism class of a $\mathbb{G}_{a, S}$-torsor $f: P \rightarrow S$ in $H_{\text {èt }}^{1}\left(S, \mathbb{G}_{a, S}\right) \cong H_{\text {èt }}^{1}\left(S, \mathcal{O}_{S}\right)$ coincides via the isomorphism $H_{\text {èt }}^{1}\left(S, \mathcal{O}_{S}\right) \cong \operatorname{Ext}_{\text {ét }}^{1}\left(\mathcal{O}_{S}, \mathcal{O}_{S}\right)$ with the class of the dual of the extension (1.4) in Proposition 12.

Example 14. (See also [9, Proposition 1.2] and [18, Proposition 1]). Let $\left(S^{\prime}, o\right)$ be a pair consisting of the spectrum of 2-dimensional regular local ring and its closed point $o$, and let $\rho: P \rightarrow S=S^{\prime} \backslash\{o\}$ be a $\mathbb{G}_{a, S}$-torsor. Let $\left\{\mathcal{F}_{n}\right\}_{n>0}$ be the ascending filtration of $\mathcal{A}=\rho_{*} \mathcal{O}_{P}$ associated to the $\mathbb{G}_{a, S}$-action $\mu_{P}: \mathbb{G}_{a, S} \times{ }_{S} P \rightarrow P$ on $P$. By Proposition $12, \mathcal{F}_{1}$ is a locally free sheaf of rank 2 on $S$, which is in fact free by virtue of [19, Corollary 4.1.1]. The Rees algebra $\mathcal{R}(P, \mu)=\bigoplus_{n \geq 0} \mathcal{F}_{n}$ is thus isomorphic to the polynomial ring algebra $\mathcal{O}_{S}[u, v]$ in two variables $u, v$ over $\mathcal{O}_{S}$. The surjection $D^{(1)}: \mathcal{F}_{1} \rightarrow \mathcal{F}_{0}=\mathcal{O}_{S}$ maps $u$ and $v$ to respective elements $x$ and $y$ of $\Gamma\left(S, \mathcal{O}_{S}\right)=\Gamma\left(S^{\prime}, \mathcal{O}_{S^{\prime}}\right)$, which have the property that $\left.(x, y) \mathcal{O}_{S^{\prime}}\right|_{S}=\mathcal{O}_{S}$, and the image of the open immersion

$$
j: P \hookrightarrow \operatorname{Proj}_{S}(\mathcal{R}(P, \mu)) \cong \operatorname{Proj}_{S}\left(\mathcal{O}_{S}[u, v]\right) \cong S \times_{\mathbb{Z}} \mathbb{P}_{\mathbb{Z}}^{1}
$$

is equal to the complement of the Cartier divisor $B$ with equation $x v-y u=0$. Letting $B^{\prime}$ be the closure of $B$ in $S^{\prime} \times_{\mathbb{Z}} \mathbb{P}_{\mathbb{Z}}^{1}$ we have the following alternative:

1) Either $B^{\prime}$ fully contains the fiber of $\operatorname{pr}_{S^{\prime}}: S^{\prime} \times_{\mathbb{Z}} \mathbb{P}_{\mathbb{Z}}^{1} \rightarrow S^{\prime}$ over the closed point $o$ and then $P \cong$ $S^{\prime} \times_{\mathbb{Z}} \mathbb{P}_{\mathbb{Z}}^{1} \backslash B^{\prime}$ is a nontrivial $\mathbb{G}_{a, S^{-}}$-torsor, isomorphic to the closed subscheme of $S^{\prime} \times_{\mathbb{Z}} \mathbb{A}_{\mathbb{Z}}^{2}$ with equation $x v-y u=1$,

2) Or $B^{\prime}$ extends to a section of $\operatorname{pr}_{S^{\prime}}: S^{\prime} \times_{\mathbb{Z}} \mathbb{P}_{\mathbb{Z}}^{1} \rightarrow S^{\prime}$ and then $S^{\prime} \times_{\mathbb{Z}} \mathbb{P}_{\mathbb{Z}}^{1} \backslash B^{\prime} \cong S^{\prime} \times_{\mathbb{Z}} \mathbb{A}_{\mathbb{Z}}^{1}$ and

$$
P \cong S^{\prime} \times_{\mathbb{Z}} \mathbb{P}_{\mathbb{Z}}^{1} \backslash\left(B^{\prime} \cup \operatorname{pr}_{S^{\prime}}^{-1}(o)\right) \cong S \times_{\mathbb{Z}} \mathbb{A}_{\mathbb{Z}}^{1}
$$

is the trivial $\mathbb{G}_{a, S}$-torsor.

\section{REES ALGEBRAS OF AFFINE $\mathbb{G}_{a}$-VARIETIES OVER A FIELD OF CHARACTERISTIC ZERO}

This section is devoted to the study of Rees algebras in the "absolute" case where the base scheme $S$ is the spectrum of a field $k$, which we further assume to be of characteristic zero for simplicity. We establish basic additional properties of Rees algebras in this context, with a special emphasis on their behavior with respect to equivariant birational morphisms such as the normalization or equivariant affine modifications. We also study the problem of finite generation of Rees algebras from both algebraic and geometric viewpoints. Throughout this section, we denote the additive group $\mathbb{G}_{a, k}$ simply by $\mathbb{G}_{a}$.

\subsection{Basic properties of global Rees algebras of affine $\mathbb{G}_{a}$-varieties.}

Here $S=\operatorname{Spec}(k)$ is the spectrum of an algebraically closed field $k$ of characteristic zero and $X=\operatorname{Spec}(A)$ is the spectrum of an integral $k$-algebra of finite type. In this context, a $k$-LFIHD $D=\left\{D^{(i)}\right\}_{i \geq 0}$ of $A$ is uniquely determined by $D^{(i)}=\frac{1}{i !} \partial^{i}$ where $\partial=D^{(1)}: A \rightarrow A$ is a $k$-derivation of $A$ such that $A=$ $\bigcup_{n \geq 0}\left(\bigcap_{i>n} \operatorname{Ker}^{i}\right)$. Since for every $n \geq 0$, Ker $\partial^{n} \subset \operatorname{Ker}^{i}$ for every $i \geq n$, we have in fact $A=$ $\bigcup_{i \geq 0} \operatorname{Ker} \partial^{i}$, i.e. $\partial$ is a locally nilpotent $k$-derivation of $A$ in the sense of [15]. Furthermore, the associated 
ascending filtration of $A$ consists simply of the $k$-vector subspaces $F_{n}=\operatorname{Ker} \partial^{n+1}, n \geq 0$. The subspaces $F_{n}$, which have the natural additional structure of modules over the ring $F_{0}=A_{0}=\operatorname{Ker} \partial$ of $\mathbb{G}_{a}$-invariants are called the degree modules associated to $\partial$ in $[14,15]$.

The Rees algebra of an affine $k$-variety $X=\operatorname{Spec}(A)$ with a $\mathbb{G}_{a}$-action determined by a locally nilpotent $k$-derivation $\partial$ of $A$ is thus equal to the graded algebra

$$
R(A, \partial)=\bigoplus_{n \geq 0} F_{n}=\bigoplus_{n \geq 0} \operatorname{Ker}^{n+1} .
$$

We denote by $\operatorname{gr}_{\partial} A$ the associated graded algebra $\bigoplus_{n>0} F_{n} / F_{n-1}$, where by convention $F_{-1}=\{0\}$. The locally nilpotent $k$-derivation $\partial$ induces a canonical homogeneous locally nilpotent $k$-derivation $R(\partial)$ of $R(A, \partial)$ of degree -1 given in restriction on each homogeneous component by

$$
\left.R(\partial)\right|_{F_{n}}=\partial: F_{n}=\operatorname{Ker}^{n+1} \rightarrow F_{n-1}=\operatorname{Ker}^{n}, \quad n \geq 0 .
$$

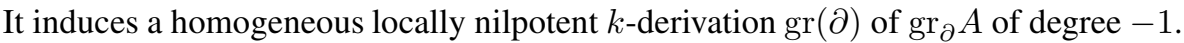

As in subsection 1.2, we can view $R(A, \partial)$ as the graded $A_{0}[\theta]$-subalgebra $\bigoplus_{n>0} F_{n} \theta^{n}$ of $A[\theta]$. It follows in particular that $R(A, \partial)$ is an integral $k$-algebra. The locally nilpotent $k[\theta]$-derivation $\theta R(\partial)$ of $R(A, \partial)$ then coincides with the restriction to $R(A, \partial)$ of the homogeneous locally nilpotent $k[\theta]$-derivation $\tilde{\partial}$ of $A[\theta]$ of degree 0 defined by $\tilde{\partial}\left(\sum a_{i} \theta^{i}\right)=\sum \partial\left(a_{i}\right) \theta^{i}$.

Lemma 15. Let $(A, \partial)$ be a finitely generated $k$-algebra endowed with a locally nilpotent $k$-derivation $\partial$ and let $R(A, \partial)$ be its Rees algebra. Then

$$
R(A, \partial)\left[\theta^{-1}\right]=R(A, \partial) \otimes_{k[\theta]} k\left[\theta^{ \pm 1}\right] \cong A \otimes_{k} k\left[\theta^{ \pm 1}\right]=A\left[\theta^{ \pm 1}\right]
$$

and the induced $k\left[\theta^{ \pm 1}\right]$-derivations $\theta R(\partial)$ and $\tilde{\partial}$ of $R(A, \partial)\left[\theta^{ \pm 1}\right]$ and $A\left[\theta^{ \pm 1}\right]$ respectively coincide under this isomorphism.

Proof. The inclusion $R(A, \partial)\left[\theta^{-1}\right] \subseteq A\left[\theta^{ \pm 1}\right]$ is clear. Conversely, let $x=\theta^{-k}\left(a_{0}+a_{1} \theta+\cdots a_{n} \theta^{n}\right) \in$ $A\left[\theta^{ \pm 1}\right]$. Since $A=\bigcup_{m \geq 1} \operatorname{Ker}^{m}$, there exists $m_{0} \geq 1$ such that $a_{i} \in \operatorname{Ker}^{m_{0}}$ for every $i$. It follows that $a_{i} \theta^{i}=\theta^{i-m_{0}}\left(a_{i} \theta^{m_{0}}\right) \in R(A, \partial)\left[\theta^{-1}\right]$ and then that

$$
\begin{aligned}
x & =\theta^{-k}\left(\theta^{-m_{0}}\left(a_{0} \theta^{m_{0}}\right)+\cdots \theta^{n-m_{0}}\left(a_{n} \theta^{m_{0}}\right)\right) \\
& =\theta^{-k-m_{0}}\left(a_{0} \theta^{m_{0}}+a_{1} \theta^{m_{0}+1}+\cdots a_{n} \theta^{m_{0}+n}\right)
\end{aligned}
$$

where for every $i, a_{i} \theta^{m_{0}+i} \in\left(\operatorname{Ker} \partial^{m_{0}+i}\right) \theta^{m_{0}+i}$ since $a_{i} \in \operatorname{Ker}^{m_{0}} \subset \operatorname{Ker}^{m_{0}+i}$. Thus $A\left[\theta^{ \pm 1}\right] \subseteq$ $R(A, \partial)\left[\theta^{-1}\right]$. The fact that the induced derivations coincide follows by construction.

Lemma 16. Let $(A, \partial)$ be an integral $k$-algebra endowed with a locally nilpotent $k$-derivation $\partial$ and let $\left\{F_{n}\right\}_{n \geq 0}$ be the associated ascending filtration of $A$. Then for every $s \in F_{1} \backslash F_{0}$, there exists an isomorphism of graded algebras

$$
\left(R(A, \partial)_{\partial s}, R(\partial)\right) \cong\left(R\left(\left(F_{0}\right)_{\partial s}[s], \frac{\partial}{\partial s}\right), R\left(\frac{\partial}{\partial s}\right)\right)
$$

where $\partial s \in F_{0}$ is viewed as homogeneous element of degree 0 in $R(A, \partial)$.

Proof. Since $\partial s \in F_{0}$, it belongs to $\operatorname{Ker} R(\partial)$. Thus $R(\partial)$ extends in a canonical way to a homogeneous locally nilpotent $k$-derivation of $R(A, \partial)_{\partial s}=R\left(A_{\partial s}, \partial\right)$ which we denote by the same symbol. On the other hand, the $A_{0}$-subalgebra $A_{0}[s]$ of $A$ generated by $s$ is stable under $\partial$, and $\partial$ restricts on $A_{0}[s]$ to the nonzero locally nilpotent $A_{0}$-derivation $\frac{\partial}{\partial s}$. Since $\partial s \in A_{0}, \frac{\partial}{\partial s}$ and $\partial$ extend to well-defined locally nilpotent $k$-derivations of the localizations $A_{0}[s]_{\partial s}=\left(A_{0}\right)_{\partial s}[s]$ and $A_{\partial s}$ respectively, which we denote again by the same symbol. By [15, Principle 11 (d)], the inclusion $\left(A_{0}[s], \frac{\partial}{\partial s}\right) \subset(A, \partial)$ induces an isomorphism $\left(\left(A_{0}\right)_{\partial s}[s], \frac{\partial}{\partial s}\right) \cong\left(A_{\partial s}, \partial\right)$. This in turns induces the desired isomorphism $R\left(\left(A_{0}\right)_{\partial s}[s], \frac{\partial}{\partial s}\right) \cong R\left(A_{\partial s}, \partial\right)$ for which $R(\partial)$ coincides with $R\left(\frac{\partial}{\partial s}\right)$. 
Remark 17. In the setting of Lemma 16, it follows in turn from Proposition 12 that $R(A, \partial)_{\partial s}$ is canonically isomorphic to the symmetric algebra of the free $\left(F_{0}\right)_{\partial s}$-submodule $F_{1} A_{\partial s} \cong\left(F_{0}\right)_{\partial s} \cdot s \oplus\left(F_{0}\right)_{\partial s}$ of rank 2 of $A_{\partial s}$. This yields an isomorphism of graded algebras

$$
\left(R(A, \partial)_{\partial s}, R(\partial)\right) \cong\left(\left(F_{0}\right)_{\partial s}[s, \theta], \frac{\partial}{\partial s}\right)
$$

where $s$ and $\theta$ are homogeneous of degree 1 .

2.2. Rees algebras and equivariant birational morphisms. We now consider the behavior of Rees algebras under certain equivariant birational morphisms between affine varieties. Let $(A, \partial)$ be an integral $k$-algebra endowed with a non-zero locally nilpotent $k$-derivation and let $A^{\prime} \subset \operatorname{Frac}(A)$ be its normalization, i.e. its integral closure in its field of fraction $\operatorname{Frac}(A)$. By results of Seidenberg and Vasconcelos (see e.g. [13, Proposition 1.2.15 and Proposition 1.3.37]), there exists a unique extension of $\partial$ to a locally nilpotent $k$-derivation $\partial^{\prime}$ of $A^{\prime}$.

Lemma 18. With the above notation, the Rees algebra $R\left(A^{\prime}, \partial^{\prime}\right)$ is the normalization of the Rees algebra $R(A, \partial)$. Furthermore, the unique extension to $R\left(A^{\prime}, \partial^{\prime}\right)$ of the canonical homogeneous locally nilpotent $k$ derivation $R(\partial)$ of $R(A, \partial)$ coincides with the canonical homogeneous locally nilpotent $k$-derivation $R\left(\partial^{\prime}\right)$ of $R\left(A^{\prime}, \partial^{\prime}\right)$.

Proof. Let $\left\{F_{n}\right\}_{n \geq 0}$ and $\left\{F_{n}^{\prime}\right\}_{n \geq 0}$ be the ascending filtrations of $A$ and $A^{\prime}$ associated to $\partial$ and $\partial^{\prime}$ respectively. Let $R=\bigoplus_{n \geq 0} F_{n} \theta^{n} \cong R(A, \partial)$ and $R^{\prime}=\bigoplus_{n \geq 0} F_{n}^{\prime} \theta^{n} \cong R\left(A^{\prime}, \partial^{\prime}\right)$. Since $F_{n}=F_{n}^{\prime} \cap A$ by construction of $\partial^{\prime}$, we have the following commutative diagram of inclusions

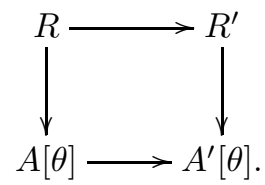

By Lemma 15 , we have $R\left[\theta^{-1}\right]=A\left[\theta^{ \pm 1}\right]$ and $R^{\prime}\left[\theta^{-1}\right]=A^{\prime}\left[\theta^{ \pm 1}\right]$, so that $R$ and $R^{\prime}$ have the same field of fractions. The normalization of $R$ is thus contained in that of $R^{\prime}$, and since on the other hand every homogeneous element $x^{\prime} \in F_{n}^{\prime} \subset A^{\prime}$ is integral over $A, R^{\prime}$ is contained in the normalization of $R$. It is thus enough to show that $R^{\prime}$ is normal. If $h \in \operatorname{Frac}\left(R^{\prime}\right) \cong \operatorname{Frac}\left(A^{\prime}\right)(\theta)$ is integral over $R^{\prime}$ then it is also integral over $R^{\prime}\left[\theta^{-1}\right] \cong A^{\prime}\left[\theta^{ \pm 1}\right]$, hence belongs to this algebra, $A^{\prime}\left[\theta^{ \pm 1}\right]$ being normal as $A^{\prime}$ is normal. It follows that $h=\theta^{-\ell} g$ for some $g \in A^{\prime}[\theta]$ which is integral over $R^{\prime}$, and it remains to prove that $R^{\prime}$ is integrally closed in $A^{\prime}[\theta]$.

Since the inclusion $R^{\prime} \hookrightarrow A^{\prime}[\theta]$ is a graded homomorphism, the integral closure of $R^{\prime}$ in $A^{\prime}[\theta]$ is a graded $R^{\prime}$-subalgebra of $A^{\prime}[\theta]$ [4, $\$ 1.8$ Proposition 20]. As a consequence, $R^{\prime}$ is integrally closed in $A^{\prime}[\theta]$ if and only if every homogeneous element $g=h \theta^{n} \in A^{\prime}[\theta]$ which is integral over $R^{\prime}$ belongs to $R^{\prime}$. Let $g^{m}+\sum_{i=0}^{m-1} a_{i} g^{i}=0$ be a homogeneous integral dependence relation with coefficients in $R^{\prime}$. Since $g^{i}=h^{i} \theta^{n i}, a_{i}$ is homogeneous of degree $(m-i) n$, hence is of the form $b_{(m-i) n} \theta^{(m-i) n}$ for some $b_{(m-i) n} \in$ $F_{(m-i) n}^{\prime}$. This implies that the relation $h^{m}+\sum_{i=0}^{m-1} b_{(m-i) n} h^{i}=0$ holds in $A^{\prime}$. If $n=0$, then $h \in A^{\prime}$ is integral over $A_{0}^{\prime}$, hence belongs to $A_{0}^{\prime}$ since the latter is integrally closed in $A^{\prime}$ by [15, Proposition 1.13]. If $n \geq 1$, then by definition $g \in R^{\prime}$ if and only if $h \in F_{n}^{\prime}$. So suppose that $h \in F_{d}^{\prime} \backslash F_{d-1}^{\prime}$ for some $d>n$. Then $h^{m} \in F_{m d}^{\prime} \backslash F_{m d-1}^{\prime}$ but on the other hand $\sum_{i=0}^{m-1} b_{(m-i) n} h^{i} \in F_{d m-1}^{\prime}$ as $F_{(m-i) n+d i}^{\prime}=F_{m n+(d-n) i}^{\prime}$ is contained $F_{d m-1}^{\prime}$ for every $i=0, \ldots, m-1$. This is absurd, so $h \in F_{n}^{\prime}$ and then $g \in R^{\prime}$.

Corollary 19. Let $A$ be an integral normal $k$-algebra. Then for every nonzero locally nilpotent $k$-derivation $\partial$ of $A$, the Rees algebra $R(A, \partial)$ is integral and normal.

Let $(A, \partial)$ be an integral $k$-algebra endowed with a non-zero locally nilpotent $k$-derivation, let $I \subset A$ be a $\partial$-invariant ideal and $f \in I$ be a $\partial$-invariant element, so that $\partial f=0$ by [15, Corollary 1.23]. Let $\tilde{\partial}$ be the locally nilpotent $k[t]$-derivation of $A[t]$ of degree 0 defined by $\tilde{\partial}\left(\sum a_{i} t^{i}\right)=\sum \partial\left(a_{i}\right) t^{i}$ and let $\bar{\partial}$ be the 
locally nilpotent $k$-derivation of $A[t] /(1-f t)$ that it induces. Since $\partial I \subset I, \bar{\partial}$ restricts to a locally nilpotent $k$-derivation of the integral $k$-algebra

$$
A[I / f]=\left(\bigoplus_{n \geq 0} I^{n} t^{n}\right) /(1-f t) \subseteq A[t] /(1-f t) \cong A\left[f^{-1}\right]
$$

which we denote by $\partial^{\prime}$. The natural inclusion $A \hookrightarrow A[I / f]$ induces an isomorphism of $k$-algebras $A\left[f^{-1}\right] \cong$ $A[I / f]\left[f^{-1}\right]$. Furthermore, $A$ is a $\partial^{\prime}$-invariant subalgebra of $A[I / f]$ and the restriction of $\partial^{\prime}$ to $A$ is equal to $\partial$. Following [23], we call the pair $\left(A[I / f], \partial^{\prime}\right)$ the equivariant affine modification of $(A, \partial)$ with center at the $\partial$-invariant ideal $I$ and $\partial$-invariant divisor $f$.

Lemma 20. With the above notation, the pair $\left(R\left(A[I / f], \partial^{\prime}\right), R\left(\partial^{\prime}\right)\right)$ is isomorphic to the equivariant affine modification $\left(R(A, \partial)[J / f], R(\partial)^{\prime}\right)$ of $(R(A, \partial), R(\partial))$ with center at the $R(\partial)$-invariant homogeneous ideal $J \subset R(A, \partial)$ generated by the elements of $I$ and with $R(\partial)$-invariant divisor $f$.

Proof. Every element $h$ of $J$ is a finite sum $h=\sum h_{i} f_{i}$ where $h_{i} \in I$ and

$$
f_{i}=\sum f_{i j} \in \bigoplus_{n \geq 0} F_{n}=R(A, \partial)
$$

Since $A=\bigcup_{n \geq 0} F_{n}$, each $h_{i}$ is homogeneous of a certain degree when viewed as an element of $R(A, \partial)$. Since $h_{i} f_{i j} \in \bar{I}$ for every $i, j$ and $I$ is $\partial$-stable, it follows that $J$ is an $R(\partial)$-stable homogeneous ideal of $R(A, \partial)$. Viewing $f$ as a homogeneous element of degree 0 in $R\left(A[I / f], \partial^{\prime}\right)$, the image of $R\left(A[I / f], \partial^{\prime}\right)$ by the injective homogeneous localization homomorphism

$$
R\left(A[I / f], \partial^{\prime}\right) \rightarrow R\left(A[I / f], \partial^{\prime}\right)\left[f^{-1}\right]=R\left(A_{f}, \partial^{\prime}\right)=R\left(A_{f}, \partial\right)=R(A, \partial)_{f}
$$

is equal to the graded subalgebra of $R\left(A_{f}, \partial\right)$ whose elements have the form $f^{-\ell} \sum g_{i}$ where $\sum g_{i} \in$ $I^{\ell} R(A, \partial)=J^{\ell}$. On the other hand, it follows from the definition of $R(A, \partial)[J / f]$ that this sub-algebra is the image of $R(A, \partial)[J / f]$ by the injective homogeneous localization homomorphism

$$
R(A, \partial)[J / f] \rightarrow R(A, \partial)[J / f]\left[f^{-1}\right]=R(A, \partial)_{f} .
$$

The equivariance then follows readily from the construction of the $k$-derivations $R\left(\partial^{\prime}\right)$ and $R(\partial)^{\prime}$.

Corollary 21. Let $A$ be a finitely generated $k$-algebra endowed with a nonzero locally nilpotent $k$-derivation $\partial$. If $R(A, \partial)$ is a finitely generated $k$-algebra, then so is $\left.R(A[I / f]), \partial^{\prime}\right)$ for every equivariant affine modification $A[I / f]$ of $A$.

Proof. Indeed, if $R(A, \partial)$ is of finite type over $k$, then $J=I R(A, \partial)$ is a finitely generated ideal, which implies in turn that $R\left(A[I / f], \partial^{\prime}\right) \cong(R(A, \partial)[J / f]$ is of finite type over $k$.

2.3. Finitely generated Rees algebras. It is well known that the ring of invariants of a $\mathbb{G}_{a}$-action on an affine $k$-variety $X=\operatorname{Spec}(A)$ is in general not finitely generated (see e.g. [15, Chapter 7] for a survey). As a consequence, the Rees algebra $R(A, \partial)$ as well as the associated graded algebra $\operatorname{gr}_{\partial} A$ of an integral $k$-algebra of finite type $A$ endowed with a non-zero locally nilpotent $k$-derivation are in general not finitely generated. Our aim in this subsection is to give an algebro-geometric construction of all pairs $(A, \partial)$ consisting of a $k$-algebra of finite type and a locally nilpotent $k$-derivation of $A$ for which the Rees algebra $(A, \partial)$ is finitely generated. Since normalization is a finite morphism, it follows from the Artin-Tate lemma that a $k$-algebra is finitely generated if and only its normalization is finitely generated. By Lemma 18, we can thus restrict without loss of generality to the case of normal $k$-algebras.

Lemma 22. Let $(A, \partial)$ be an integral normal $k$-algebra of finite type endowed with a locally nilpotent $k$ derivation $\partial$, let $\left\{F_{n}\right\}_{n \geq 0}$ be the associated ascending filtration and let $R(A, \partial)=\bigoplus_{n \geq 0} F_{n}$ be its Rees algebra. Then the following are equivalent:

1) The algebra $R(A, \partial)$ is finitely generated over $k$,

2) The associated graded algebra $\operatorname{gr}_{\partial} A$ is finitely generated over $k$,

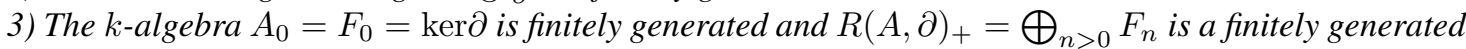
$R(A, \partial)$-module. 
Proof. As in (1.2), we identify $R(A, \partial)$ with the graded sub- $A_{0}$-algebra $\bigoplus_{n>0} F_{n} \theta^{n}$ of $A[\theta]$. The implication 3$) \Rightarrow 1$ ) is straightforward and the implication 1) $\Rightarrow 2$ ) follows immediately from the definition of $\operatorname{gr}_{\partial} A=R(A, \partial) / \theta R(A, \partial)$. To show the implication 2) $\Rightarrow 3$ ), we can assume without loss of generality that $\operatorname{gr}_{\partial} A=k\left[b_{1}, \ldots, b_{r}\right]$ for some nonzero homogeneous elements $b_{i} \in F_{d(i)} / F_{d(i)-1}, i=1, \ldots, r$, where $d(i)=0$ for $i=1, \ldots, m$ and $d(i)>0$ for $i=m+1, \ldots, r$. It follows in particular that $F_{0}=F_{0} / F_{-1}$ is generated by $b_{1}, \ldots, b_{m}$. Choosing representatives $a_{i} \in F_{d(i)} \backslash F_{d(i)-1}$ of the classes $b_{i}$, we have $F_{0}=k\left[b_{1}, \ldots, b_{m}\right]=k\left[a_{1}, \ldots, a_{m}\right]$. We claim that $R(A, \partial)_{+}$is equal to the homogeneous ideal $I$ generated by $\theta$ and the elements $a_{i} \theta^{d(i)}, i=m+1, \ldots, r$. Indeed, let $f \theta^{d} \in F_{d} \theta^{d} \subset R(A, \partial)_{+}$be a homogeneous element and let $d_{0}$ be minimal such that $f \in F_{d_{0}} \backslash F_{d_{0}-1}$. If $d_{0}<d$ then $f \theta^{d_{0}} \in F_{d_{0}} \theta^{d_{0}} \subset R(A, \partial)$ and then $f \theta^{d}=\left(f \theta^{d_{0}}\right) \theta^{d-d_{0}} \in I$. Otherwise, if $d_{0}=d$, the residue class $\bar{f}$ of $f$ in $F_{d} / F_{d-1}$ is nonzero, and by hypothesis, there exists a homogeneous polynomial $P \in F_{0}\left[t_{m+1}, \ldots, t_{r}\right]$ of degree $d$ with respect to the weights $d\left(t_{i}\right)=d(i), i=m+1, \ldots, r$, such that $\bar{f}=P\left(b_{m+1}, \ldots, b_{r}\right)$. It follows that

$$
f \theta^{d}-P\left(a_{m+1} \theta^{d(m+1)}, \ldots, a_{r} \theta^{d(r)}\right) \in F_{d} \theta^{d}
$$

is contained in the subspace $F_{d-1} \theta^{d}$, hence is equal to $\left(g_{d-1} \theta^{d-1}\right) \theta$ for some element $g_{d-1} \theta^{d-1} \in F_{d-1} \theta^{d-1}$ of $R(A, \partial)$, which implies in turn that $f \theta^{d}$ belongs to $I$.

2.3.1. Geometric criterion for finite generation. Recall that a $\mathbb{P}^{1}$-fibration between algebraic $k$-varieties is a surjective projective morphism of finite type $\pi: Y \rightarrow Y_{0}$ whose fiber $Y_{\eta}$ over the generic point $\eta$ of $Y_{0}$ is isomorphic to the projective line $\mathbb{P}_{k\left(Y_{0}\right)}^{1}$ over the field of rational functions $k\left(Y_{0}\right)$ of $Y_{0}$.

Proposition 23. Let $(A, \partial)$ be an integral normal $k$-algebra of finite type endowed with a nontrivial locally nilpotent $k$-derivation $\partial$ whose Rees algebra $R(A, \partial)=\bigoplus_{n \geq 0} F_{n} \theta^{n} \subseteq A[\theta]$ is a finitely generated $k$ algebra. Let $A_{0}=F_{0}$ and let

$$
X=\operatorname{Spec}(A) \hookrightarrow Y=\operatorname{Proj}_{k}(R(A, \partial))
$$

be the open embedding of schemes over $Y_{0}=\operatorname{Spec}\left(A_{0}\right)$ induced by the graded inclusion $\eta: R(A, \partial) \hookrightarrow A[\theta]$ (see (1.2)). Then the following hold:

1) The schemes $Y_{0}$ and $Y$ are normal $k$-varieties,

2) The structure morphism $\pi: Y \rightarrow Y_{0}$ is a $\mathbb{P}^{1}$-fibration,

3) The effective Weil divisor $B=V_{+}(\theta)$ on $Y$ is ample and the restriction of the sheaf $\mathcal{O}_{Y}(B)$ to the generic fiber $Y_{\eta} \cong \mathbb{P}_{k\left(Y_{0}\right)}^{1}$ of $\pi$ is equal to $\mathcal{O}_{\mathbb{P}_{k\left(Y_{0}\right)}^{1}}(1)$.

Proof. By Lemma 22, $A_{0}$ is a $k$-algebra of finite type. Since $A$ is normal by assumption, the normality of $Y_{0}=\operatorname{Spec}\left(A_{0}\right)$ and $Y$ follow from [15, Proposition 1.13] and Lemma 18 respectively. Since $R(A, \partial)$ is finitely generated over $A_{0}$, it follows from [16, Proposition 4.6.18] that $\pi: Y=\operatorname{Proj}_{k}(R(A, \partial)) \rightarrow Y_{0}$ is a morphism of finite type and that there exists $d \geq 1$ such that the quasi-coherent $\mathcal{O}_{Y}$-module $\mathcal{O}_{Y}(d)$ associated to $F_{d}$ is invertible and $\pi$-ample. It follows that $d B=V_{+}\left(\theta^{d}\right)$ is a $\pi$-ample Cartier divisor, and since $Y_{0}$ is affine, we deduce in turn from [16, Proposition 4.5.10] that $B$ is $\mathbb{Q}$-Cartier and ample on $Y$. By Lemma 6, the image of the open embedding $X \hookrightarrow Y$ coincides with the complement of the support of $B$ on $Y$. Furthermore, by Lemma 8, the inclusion $X \hookrightarrow Y$ is equivariant for the $\mathbb{G}_{a}$-actions associated with the locally nilpotent $k$-derivations $\partial$ and $\theta R(\partial)$ on $A$ and $R(A, \partial)$ respectively. Lemma 16 implies that for every $s \in F_{1} \backslash F_{0}$, we have an isomophism

$$
\left(R(A, \partial)_{\partial s}, \theta R(\partial)\right) \cong\left(\left(A_{0}\right)_{\partial s}[s, \theta], \theta \frac{\partial}{\partial s}\right) .
$$

It follows that the restriction of $\pi$ over the principal affine open subset $\left(Y_{0}\right)_{\partial s} \cong \operatorname{Spec}\left(\left(A_{0}\right)_{\partial s}\right)$ is isomorphic to the trivial $\mathbb{P}^{1}$-bundle $\operatorname{Proj}_{k}\left(\left(A_{0}\right)_{\partial s}[s, \theta]\right) \rightarrow\left(Y_{0}\right)_{\partial s}$ and that the restriction of $\mathcal{O}_{Y}(B)$ over $\left(Y_{0}\right)_{\partial s}$ is equal to $\mathcal{O}_{\mathbb{P}_{\left(A_{0}\right)_{\partial s}}^{1}}(1)$.

Conversely, given a normal affine $k$-variety $Y_{0}$ and a $\mathbb{P}^{1}$-fibration $\pi: Y \rightarrow Y_{0}$ where $Y$ is a normal $k$ variety, it is a natural question to characterize which effective Weil divisors $B$ on $Y$ have the property that their complements are affine varieties carrying $\mathbb{G}_{a}$-actions with finitely generated associated Rees algebras. 
Recall that a Weil divisor $B$ on a $k$-variety $Y$ is called semi-ample if there exists $n \geq 1$ such that the sheaf $\mathcal{O}_{Y}(n B)$ is invertible and generated by its global sections. We then have the following criterion:

Theorem 24. Let $Y_{0}=\operatorname{Spec}\left(A_{0}\right)$ be a normal affine $k$-variety and let $\pi: Y \rightarrow Y_{0}$ be a $\mathbb{P}^{1}$-fibration where $Y$ is a normal $k$-variety. Let $B$ an effective semi-ample Weil divisor on $Y$ with the following properties:

a) The scheme $X=Y \backslash B$ is an affine $k$-variety,

b) The restriction of the sheaf $\mathcal{O}_{Y}(B)$ to the generic fiber $Y_{\eta} \cong \mathbb{P}_{k\left(Y_{0}\right)}^{1}$ of $\pi$ is equal to $\mathcal{O}_{\mathbb{P}_{k\left(Y_{0}\right)}^{1}}$ (1).

Then the following hold:

1) There exists a nontrivial $\mathbb{G}_{a, Y_{0}}$-action on $Y$ which leaves $B$ invariant and restricts to a $\mathbb{G}_{a, Y_{0}}$-action on $X$.

2) The Rees algebra $R(A, \partial)$ of the locally nilpotent $k$-derivation $\partial$ of $A=\Gamma\left(X, \mathcal{O}_{X}\right)$ corresponding to the induced $\mathbb{G}_{a, Y_{0}}$-action on $X$ is a finitely generated $A_{0}$-algebra isomorphic to $R(Y, B)=\bigoplus_{n \geq 0} H^{0}\left(Y, \mathcal{O}_{Y}(n B)\right)$.

Proof. By definition, $\mathcal{L}=\mathcal{O}_{Y}(B)$ is the reflexive subsheaf of rank 1 of the constant sheaf $\mathcal{K}_{Y}$ of rational functions on $Y$ defined by

$$
\mathcal{L}(U)=\left\{f \in \mathcal{K}_{Y}(U), \operatorname{div}(f)+\left.B\right|_{U} \geq 0\right\} \cup\{0\}
$$

for every open subset $U$ of $Y$. The fact that $B$ is effective implies that the constant section 1 of $\mathcal{K}_{Y}$ is contained in $\mathcal{L}$. We denote by $\theta \in H^{0}(Y, \mathcal{L})$ the corresponding global section of $\mathcal{L}$ whose zero locus is equal to $B$. We then get an inclusion

$$
H^{0}\left(Y, \mathcal{O}_{Y}\right) \hookrightarrow H^{0}(Y, \mathcal{L}), f \mapsto f \theta .
$$

Since $Y$ is projective over the affine variety $Y_{0}$, by [17, Chapter III,Theorem 5.2], we have $H^{0}\left(Y, \mathcal{O}_{Y}\right)=A_{0}$ and $H^{0}\left(Y, \mathcal{L}^{\otimes n}\right)$ is a finitely generated $A_{0}$-module for every $n$. Furthermore, the restriction homomorphism

$$
H^{0}\left(Y, \mathcal{L}^{\otimes n}\right) \rightarrow H^{0}\left(Y_{\eta}, \mathcal{L}^{\otimes n}\right)=H^{0}\left(Y_{\eta}, \mathcal{O}_{Y_{\eta}}\left(\left.n B\right|_{Y_{\eta}}\right)\right) \cong H^{0}\left(\mathbb{P}_{k\left(Y_{0}\right)}^{1}, \mathcal{O}_{\mathbb{P}_{k\left(Y_{0}\right)}^{1}}(n)\right)
$$

is surjective. The fact that for an effective semi-ample Weil divisor $B$ the algebra $R(Y, \mathcal{L})=R(Y, B)$ is finitely generated over $A_{0}$ is a classical result due to Zariski (see e.g. [31]). Let us briefly recall the argument. Since $\mathcal{L}$ is semi-ample, it follows from [24, Theorem 2.1.27] that for sufficiently big and divisible $d \geq 1$, the sheaf $\mathcal{L}^{\otimes d}$ is invertible and the rational map

$$
\psi_{d}: Y \rightarrow \mathbb{P}\left(H^{0}\left(Y, \mathcal{L}^{\otimes d}\right)^{*}\right)
$$

is an everywhere defined morphism of $Y_{0}$-schemes with connected fibers, whose image is a normal variety $\pi_{d}: Y_{d} \rightarrow Y_{0}$ projective over $Y_{0}$, and such that we have $\mathcal{L}^{\otimes d}=\psi_{d}^{*} \mathcal{O}_{Y_{d}}(1)$. This implies that the Veronese subring $R(Y, \mathcal{L})^{(d)}=\bigoplus_{n \geq 0} H^{0}\left(Y, \mathcal{L}^{\otimes n d}\right)$ is finitely generated over $A_{0}$, and hence that $R(Y, B)=R(Y, \mathcal{L})$ is finitely generated by [3, Corollary 1.2.5].

It follows that $Y^{\prime}=\operatorname{Proj}_{k}(R(Y, B))$ is a normal variety, projective over $Y_{0}$ and that the canonical rational map of $Y_{0}$-schemes

$$
\psi: Y \rightarrow Y^{\prime}=\operatorname{Proj}_{k}(R(Y, B))
$$

is a morphism. Since by hypothesis the restriction of $\mathcal{L}$ to $Y_{\eta}$ is invertible and very ample, $\psi$ restricts to an isomorphism over the generic point $\eta$ of $Y_{0}$, hence is birational. Furthermore, since $X=Y \backslash B$ is affine hence does not contain any complete curve, it follows that $B \cdot C>0$ for every complete curve in $Y$ intersecting $X$. This implies that the restriction of $\psi$ to $Y \backslash B$ is quasi-finite and birational, hence an isomorphism onto its image by Zariski Main Theorem. The latter coincides by construction with the complement

$$
D_{+}(\theta) \cong \operatorname{Spec}(R(Y, B) /(1-\theta) R(Y, B))
$$

of the Weil divisor $B^{\prime}=V_{+}(\theta)$ on $Y^{\prime}$. Hypothesis b) implies further that there exists a global section $s \in H^{0}(Y, \mathcal{L})$ different from $\theta$ such that

$$
\operatorname{Proj}_{k}(R(Y, B)) \times_{Y_{0}} \operatorname{Spec}\left(k\left(Y_{0}\right)\right) \cong \operatorname{Proj}_{k\left(Y_{0}\right)}\left(k\left(Y_{0}\right)[s, \theta]\right) .
$$

It follows that there exists $f \in A_{0}$ such that the homogeneous locally nilpotent $k\left(Y_{0}\right)$-derivation $f \theta \frac{\partial}{\partial s}$ of degree 0 of $k\left(Y_{0}\right)[s, \theta]$ extends to a homogeneous locally nilpotent $A_{0}$-derivation $\tilde{\partial}$ of $R(Y, B)$ of degree 0 defining a $\mathbb{G}_{a, Y_{0}}$-action on $Y^{\prime}$ leaving the Weil divisor $B^{\prime}$ invariant and inducing the trivial action on $B^{\prime}$. Since 
$\psi: Y \rightarrow Y^{\prime}$ restricts to an isomorphism $Y \backslash B \rightarrow Y^{\prime} \backslash B^{\prime}$, this action lifts to a $\mathbb{G}_{a, Y_{0}}$-action on $Y$ leaving $B$ invariant and hence $X=Y \backslash B$ invariant. By construction, the Rees algebra of the associated locally nilpotent $A_{0}$-derivation $\partial$ of $\Gamma\left(X, \mathcal{O}_{X}\right)$ is isomorphic to the finitely generated algebra $R(Y, B)=R\left(Y^{\prime}, B^{\prime}\right)$.

Given a pair $\left(\pi: Y \rightarrow Y_{0}, B\right)$ satisfying the hypotheses of Theorem 24 , the proof actually shows that the composition of the open embedding $X=Y \backslash B \hookrightarrow Y$ with the canonical morphism

$$
\psi: Y \rightarrow Y^{\prime}=\operatorname{Proj}_{k}(R(Y, B))
$$

of schemes over $Y_{0}$ is an open embedding of $X$ in $Y^{\prime}$ as the complement of the ample Weil divisor $B^{\prime}=$ $\psi_{*}(B)$. The following example illustrates the fact that even when $X$ is smooth, the variety $Y^{\prime}$ can have bad singularities supported along $B^{\prime}$ so that, depending on the context, it can be more convenient to consider a model $(Y, B)$ with better singularities but non-ample boundary divisor $B$.

Example 25. Let $X \subset \mathbb{A}_{k}^{4}=\operatorname{Spec}(k[x, y, u, v])$ be the smooth affine 3 -fold with equation $x v=y(y u+1)$. The locally nilpotent $k[x, y]$-derivation

$$
\partial=x \frac{\partial}{\partial u}+y^{2} \frac{\partial}{\partial v}
$$

of the coordinate ring $A$ of $X$ defines a $\mathbb{G}_{a}$-action on $X$. The ring of invariants $A_{0}$ is equal to $k[x, y]$ and the corresponding $\mathbb{G}_{a}$-invariant morphism $\pi=\operatorname{pr}_{x, y}: X \rightarrow \mathbb{A}_{k}^{2}$ restricts to a $\mathbb{G}_{a}$-torsor over the complement of the origin $(0,0)$. On the other hand, $\pi^{-1}((0,0))$ is isomorphic to $\mathbb{A}_{k}^{2}=\operatorname{Spec}(k[u, v])$ and consists of $\mathbb{G}_{a}$-fixed points only.

The Rees algebra $R(A, \partial)$ is isomorphic to the quotient of $k[x, y][u, v, \theta]$ by the homogeneous ideal generated by $x v-y^{2} u-y \theta$, where $u, v$ and $\theta$ all have weight 1 . So $Y^{\prime}=\operatorname{Proj}_{k}(R(A, \partial))$ is isomorphic to the closed sub-variety in $\mathbb{A}_{k}^{2} \times \mathbb{P}_{k}^{2}=\operatorname{Proj}_{k[x, y]}(k[x, y][u, v, \theta])$ defined by the equation $x v-y^{2} u-y \theta=0$, and $X=Y^{\prime} \backslash B^{\prime}$ where $B^{\prime}$ is the irreducible ample relative hyperplane section $\{\theta=0\}$, isomorphic to the blow-up of $\mathbb{A}_{k}^{2}$ with center at the closed subscheme with defining ideal $\left(x, y^{2}\right)$. The projection $\bar{\pi}=\operatorname{pr}_{x, y}: Y^{\prime} \rightarrow \mathbb{A}_{k}^{2}$ restricts to a locally trivial $\mathbb{P}^{1}$-bundle over the complement of the origin whereas the fiber $\bar{\pi}^{-1}(0,0)$ is isomorphic to $\mathbb{P}_{k}^{2}=\operatorname{Proj}_{k}(k[u, v, \theta])$. The $k[x, y]$-derivation $\partial$ extends to the homogeneous $k[x, y, \theta]$-derivation $\theta \partial$ of degree 0 of $k[x, y][u, v, \theta]$ defining a $\mathbb{G}_{a}$-action

$$
((x, y),[u: v: \theta]) \mapsto\left((x, y),\left[u+t x \theta: v+y^{2} \theta: \theta\right]\right)
$$

on $\mathbb{A}_{k}^{2} \times \mathbb{P}_{k}^{2}$, leaving $Y^{\prime}$ invariant. Its restriction to $X$ is equal to that defined by $\partial$ whereas it restriction to $B^{\prime}$ is the trivial $\mathbb{G}_{a}$-action.

It is easily seen by the Jacobian criterion that $Y^{\prime}$ has a unique singular point $p=((0,0),[1: 0: 0])$, which is contained in $B^{\prime}$. Let $c: Y \rightarrow Y^{\prime}$ be the blow-up of the Weil divisor $D=\bar{\pi}^{-1}(0,0)$ and let $E$ be its exceptional locus. Since $D$ is $\mathbb{G}_{a}$-invariant, the $\mathbb{G}_{a}$-action on $Y^{\prime}$ lifts to a $\mathbb{G}_{a}$-action on $Y$. Furthermore, since $Y^{\prime} \backslash\{p\}$ is smooth, $\left.D\right|_{Y^{\prime} \backslash\{p\}}$ is a Cartier divisor, which implies that $c$ induces a $\mathbb{G}_{a}$-equivariant isomorphism between $Y^{\prime} \backslash\{p\}$ and $c^{-1}\left(Y^{\prime} \backslash\{p\}\right) \cong Y \backslash E$. In particular, $c$ induces a $\mathbb{G}_{a}$-equivariant isomorphism between $X$ and $c^{-1}(X)=Y \backslash c^{-1}(B)$.

The intersection of $Y^{\prime}$ with the affine chart $V=\{u \neq 0\}$ of $\mathbb{A}_{k}^{2} \times \mathbb{P}_{k}^{2}$ is isomorphic to the sub-variety $x v-y z=0$ in $\mathbb{A}_{k}^{4}$, where $z=y-\theta$. The point $p$ is thus a non- $\mathbb{Q}$-factorial singularity of $Y^{\prime}$, the divisor $\left.D\right|_{V}$ is not $\mathbb{Q}$-Cartier, and the blow-up $c: Y \rightarrow Y^{\prime}$ of $D$ is a small resolution of $p$ with exceptional locus $E \cong \mathbb{P}_{k}^{1}$. The threefold $Y$ is thus smooth and $B=c^{-1}\left(B^{\prime}\right)$ is a $\mathbb{G}_{a}$-invariant irreducible semi-ample Cartier divisor which is not ample, such that $Y \backslash B$ is equivariantly isomorphic to $X$.

2.3.2. The Rees algebra algorithm. Let $X=\operatorname{Spec}(A)$ be a normal affine variety endowed with a nontrivial $\mathbb{G}_{a}$-action determined by a locally nilpotent $k$-derivation $\partial$ of $A$. Let $A_{0}=F_{0}=\operatorname{Ker} \partial$ and $\left\{F_{n}\right\}_{n \geq 0}$ be the associated ascending filtration of $A$ by its $A_{0}$-submodules. In the case where $A_{0}$ is noetherian, an algorithm to compute the modules $F_{n}$ was given by Freudenburg $[14,15]$ in the form of an extension of van den Essen's kernel algorithm for a locally nilpotent derivation $[13, \S 1.4]$. In the case where the Rees algebra $R(A, \partial)$ is finitely generated, we describe below an extension of these algorithms, which computes generators of $R(A, \partial)$ from a given set of generators of $A$ as a $k$-algebra. 
As in (1.2), we identify $R(A, \partial)$ with the graded $A_{0}$-subalgebra $\bigoplus_{n>0} F_{n} \theta^{n}$ of $A[\theta]$. Let $a_{1}, \ldots, a_{m} \in A$ be a finite collection of generators of $A$ as a $k$-algebra. For every $i=1, \ldots, m$, we choose an integer $e(i)$ so that $a_{i} \in F_{e(i)}$. We obtain a graded subalgebra

$$
R_{0}=k\left[\theta,\left\{a_{i} \theta^{e(i)}\right\}_{i=1, \ldots, m}\right] \subseteq R(A, \partial) .
$$

If equality holds, we are done. Otherwise, there exists an element $a \in F_{d} \backslash F_{d-1} \subset A$, for some $d \geq 0$, such that $a \theta^{d} \in R(A, \partial) \backslash R_{0}$. Since $a \in A$, there exists a polynomial $\tilde{P} \in k\left[X_{1}, \ldots, X_{m}\right]$ such that $a=\tilde{P}\left(a_{1}, \ldots, a_{m}\right)$. Letting $P \in k\left[X_{0}, \ldots, X_{m}\right]$ be the homogenization of $\tilde{P}$ with respect to the weights $\mathbf{e}=(1, e(1), \ldots, e(m))$, we have $P\left(\theta, a_{1} \theta^{e(1)}, \ldots, a_{m} \theta^{e(m)}\right)=a \theta^{N}$ in $R(A, \partial)$ for some $N>d$. Let $N$ be minimal with the property that $a \theta^{N} \in R_{0}$ and consider the graded homomorphism

$$
\begin{aligned}
k\left[X_{0}, \ldots, X_{m}\right] & \stackrel{\phi}{\rightarrow} R(A, \partial) \\
X_{0} & \mapsto \theta \\
X_{i} & \mapsto a_{i} \theta^{e(i)}, \text { for } i \geq 1 .
\end{aligned}
$$

Since $k\left[X_{0}, \ldots, X_{m}\right]$ is noetherian, the e-homogeneous ideal $\phi^{-1}(\theta R(A, \partial)) \subset k\left[X_{0}, \ldots, X_{m}\right]$ is finitely generated, say by elements $Q_{1}, \ldots, Q_{s} \in k\left[X_{0}, \ldots, X_{m}\right]$. By definition, there exists $q_{i} \in A$ and integers $f(i)$ such that

$$
\frac{1}{\theta} Q_{i}\left(\theta, a_{1} \theta^{e(1)}, \ldots, a_{m} \theta^{e(m)}\right)=q_{i} \theta^{f(i)} \in R(A, \partial), \text { for } 1 \leq i \leq s .
$$

Since $N>d$, the polynomial $P$ belongs to $\phi^{-1}(\theta R(A, \partial))$, and it follows that $P=\sum_{i=1}^{s} P_{i} Q_{i}$ for some e-homogeneous elements $P_{i} \in k\left[X_{0}, \ldots, X_{m}\right]$. Hence

$$
a \theta^{N-1}=\sum_{i=1}^{s} q_{i} \theta^{f(i)} P_{i}\left(\theta, a_{1} \theta^{e(1)}, \ldots, a_{m} \theta^{e(m)}\right)
$$

and it follows that

$$
a \theta^{N-1} \in k\left[\theta, a_{1} \theta^{e(1)}, \ldots, a_{m} \theta^{e(m)}, q_{1} \theta^{f(1)}, \ldots, q_{s} \theta^{f(s)}\right] \subseteq R(A, \partial) .
$$

Thus by adding the generators $q_{i} \theta^{f(i)}, i=1, \ldots, s$, to the previous ones, we obtain a subalgebra $R_{1} \subset$ $R(A, \partial)$ with the property that

$$
\min \left\{N \text { such that } a \theta^{N} \in R_{1}\right\} \leq \min \left\{N \text { such that } a \theta^{N} \in R_{0}\right\}-1 .
$$

If $R(A, \partial)$ is finitely generated over $k$, say $R(A, \partial)=k\left[g_{1} \theta^{m(1)}, \ldots, g_{l} \theta^{m(l)}\right]$ with $g_{i} \in A$, then for each $g_{i}$ there exists a minimal number $N_{i}$ such that $g_{i} \theta^{N_{i}} \in R_{0} \subset R(A, \partial)$. By iterating the above procedure at most $M=\max _{1 \leq i \leq l}\left\{N_{i}-m(i)\right\}$ times, we obtain a finitely generated subalgebra $R_{M} \subseteq R(A, \partial)$ which contains all the $g_{i} \theta^{m(i)}, i=1, \ldots, l$, hence is equal to $R(A, \partial)$.

2.4. Relation between global Rees algebras and relative Rees algebras of the fixed point free locus. Let $X=\operatorname{Spec}(A)$ be a normal affine $k$-variety endowed with a nontrivial $\mathbb{G}_{a}$-action determined by a locally nilpotent $k$-derivation $\partial$ of $A$. Let $X^{\mathbb{G}_{a}}$ denote the fixed locus of this $\mathbb{G}_{a}$-action. By $[25,10.4]$ the induced $\mathbb{G}_{a}$-action $\mu$ on $Y=X \backslash X^{\mathbb{G}_{a}}$ admits a categorical quotient in the category of algebraic spaces in the form of an étale locally trivial $\mathbb{G}_{a}$-torsor $\rho: Y \rightarrow S$ over a certain algebraic $k$-space $S$. Let $\left\{\mathcal{F}_{n}\right\}_{n \geq 0}$ be the filtration of $\rho_{*} \mathcal{O}_{Y}$ associated to the locally nilpotent $\mathcal{O}_{S}$-derivation $\delta_{S}$ of $\rho_{*} \mathcal{O}_{Y}$ corresponding to the action $\mu$. By Proposition $12, \mathcal{F}_{1}$ is an étale locally free sheaf of rank 2 on $S$, and the Rees $\mathcal{O}_{S}$-algebra $\mathcal{R}(Y, \mu)$ is isomorphic to the symmetric algebra Sym $\mathcal{F}_{1}$ of $\mathcal{F}_{1}$. The relative spectrum $p: V=\operatorname{Spec}_{S}(\mathcal{R}(Y, \mu)) \rightarrow S$ is thus an étale locally trivial vector bundle of rank 2 on $S$.

Lemma 26. With the above notation, suppose that every irreducible component of the fixed locus $X^{\mathbb{G}_{a, k}}$ has codimension at least 2 in $X$. Then $R(A, \partial) \cong \Gamma\left(V, \mathcal{O}_{V}\right)$ as graded algebras. 
Proof. Since $X \backslash Y=X^{\mathbb{G}_{a}}$ has codimension at least 2 in the normal affine variety $X$, we have

$$
\Gamma\left(S, \rho_{*} \mathcal{O}_{Y}\right)=\Gamma\left(Y, \mathcal{O}_{Y}\right)=\Gamma\left(X, \mathcal{O}_{X}\right)=A .
$$

Furthermore, since $\mu$ is the restriction to $Y$ of the $\mathbb{G}_{a}$-action determined by $\partial$, for every $n \geq 0$, the subspaces $F_{n}=\operatorname{Ker} \partial^{n+1}$ of $A$ and $\Gamma\left(S, \mathcal{F}_{n}\right)$ of $\Gamma\left(S, \rho_{*} \mathcal{O}_{Y}\right)$ coincide. Indeed, by definition $\Gamma\left(S, \delta_{S}\right)$ and $\partial$ extend to the same derivation $\tilde{\partial}$ of the field of rational functions $\operatorname{Frac}(A)$ of $X$. It is clear that $F_{n} \subseteq \Gamma\left(S, \mathcal{F}_{n}\right)$ and that conversely every element $f \in \Gamma\left(S, \mathcal{F}_{n}\right)$ is a rational function on $X$, defined everywhere except maybe on $X^{\mathbb{G}_{a}}$, and with the property that $\Gamma\left(S, \delta_{S}\right)^{n+1} f=\tilde{\partial}^{n+1} f=0$. Since $X^{\mathbb{G}_{a}}$ has codimension at least 2 and $X$ is normal, $f$ is everywhere defined on $X$ and satisfies $\partial^{n+1} f=0$. So $f$ is an element of $F_{n}$. We thus obtain isomorphisms of graded algebras

$$
\begin{aligned}
R(A, \partial) & =\bigoplus_{n \geq 0} F_{n}=\bigoplus_{n \geq 0} \Gamma\left(S, \mathcal{F}_{n}\right) \cong \Gamma\left(S, \bigoplus_{n \geq 0} \mathcal{F}_{n}\right) \\
& \cong \Gamma\left(S, \bigoplus_{n \geq 0} \operatorname{Sym} \cdot \mathcal{F}_{1}\right)=\Gamma\left(S, p_{*} \mathcal{O}_{V}\right)=\Gamma\left(V, \mathcal{O}_{V}\right) .
\end{aligned}
$$

Let $\left\{F_{n}\right\}_{n \geq 0}$ be the increasing filtration of $A$ associated to $\partial$ and let $A_{0}=F_{0}=$ Ker $\partial$. Then for every $n \geq 1$, we have an exact sequence of $A_{0}$-modules

$$
0 \rightarrow A_{0} \rightarrow F_{n} \stackrel{\partial}{\longrightarrow} F_{n-1}
$$

in which the last homomorphism is in general not surjective. In contrast, for the $\mathbb{G}_{a}$-torsor $\rho: Y \rightarrow S$, the sequences of $\mathcal{O}_{S}$-module homomorphisms

$$
0 \rightarrow \mathcal{O}_{S} \rightarrow \mathcal{F}_{n} \stackrel{\delta_{S}}{\longrightarrow} \mathcal{F}_{n-1} \rightarrow 0, \quad n \geq 1,
$$

are all exact. Suppose as in Lemma 26 that each irreducible component of $X \backslash Y=X^{\mathbb{G}_{a}}$ has codimension at least two. Taking global sections over $S$ in the above exact sequence, we obtain for every $n \geq 1$ a long exact sequence

$$
0 \rightarrow \Gamma\left(S, \mathcal{O}_{S}\right)=A_{0} \rightarrow \Gamma\left(S, \mathcal{F}_{n}\right)=F_{n} \stackrel{\Gamma\left(S, \delta_{S}\right)=\partial}{\longrightarrow} \Gamma\left(S, \mathcal{F}_{n-1}\right)=F_{n-1} \stackrel{d_{1, n}}{\rightarrow} H_{\text {ét }}^{1}\left(S, \mathcal{O}_{S}\right) \rightarrow \cdots
$$

in which the coboundary homomorphism $d_{1, n}: F_{n-1} \rightarrow H_{\text {ét }}^{1}\left(S, \mathcal{O}_{S}\right)$ maps the constant section 1 to the isomorphism class of the $\mathbb{G}_{a, k}$-torsor $\rho: Y \rightarrow S$ in $H_{\text {ét }}^{1}\left(S, \mathcal{O}_{S}\right)$. This provides a cohomological interpretation of the lack surjectivity of the homomorphism $\partial: F_{n} \rightarrow F_{n-1}$ together with an identification $\operatorname{Im}\left(\left.\partial\right|_{F_{n}}\right)=$ $\operatorname{Ker}\left(d_{1, n}\right)$.

Example 27. Let

$$
\mathrm{SL}_{2}=\left\{M=\left(\begin{array}{ll}
x & u \\
y & v
\end{array}\right) \in \mathcal{M}_{2}(k), \operatorname{det} M=1\right\} \cong \operatorname{Spec}(k[x, y, u, v] /(x v-y u-1)) .
$$

The projection $f=\operatorname{pr}_{x, y}: \mathrm{SL}_{2} \rightarrow S=\mathbb{A}_{k}^{2} \backslash\{(0,0)\}$ is a $\mathbb{G}_{a}$-torsor for the $\mathbb{G}_{a, S}$-action $\mu$ defined by right multiplication with unipotent upper triangular matrices. Let $\left\{\mathcal{F}_{n}\right\}_{n>0}$ be the corresponding ascending filtration of $\mathcal{A}=f_{*} \mathcal{O}_{\mathrm{SL}_{2}}$. By Proposition 12, $\mathcal{F}_{1}$ is a locally free sheaf of rank 2 on $S$, and the Rees algebra $\mathcal{R}\left(\mathrm{SL}_{2}, \mu\right)=\bigoplus_{n \geq 0} \mathcal{F}_{n}$ is isomorphic the symmetric algebra of $\mathcal{F}_{1}$. As a consequence of [19, Corollary 4.1.1], $\mathcal{F}_{1}$ is equal to the restriction to $S$ of a locally free sheaf $\mathcal{E}$ of rank 2 on $\mathbb{A}_{k}^{2}$, and since the latter is free by virtue of [28], it follows that $\mathcal{F}_{1} \cong \mathcal{O}_{S}^{\oplus 2}$.

Explicitly, since the $\mathbb{G}_{a, S}$-torsor $f: \mathrm{SL}_{2} \rightarrow S$ becomes trivial on the cover of $S$ by the principal affine open subsets $S_{x}=\operatorname{Spec}\left(k\left[x^{ \pm 1}, y\right]\right)$ and $S_{y}=\operatorname{Spec}\left(k\left[x, y^{ \pm 1}\right]\right)$, with equivariant trivializations

$$
\left.\mathrm{SL}_{2}\right|_{S_{x}} \cong S_{x} \times \operatorname{Spec}\left(k\left[x^{-1} u\right]\right) \quad \text { and }\left.\quad \mathrm{SL}_{2}\right|_{S_{y}} \cong S_{y} \times \operatorname{Spec}\left(k\left[y^{-1} v\right]\right)
$$


it follows that the sub- $\mathcal{O}_{S}$-module $\mathcal{F}_{1}$ of $\mathcal{A}$ is the extension of $\mathcal{O}_{S}$ by itself with trivializations $\left.\mathcal{F}_{1}\right|_{S_{x}} \cong$ $\mathcal{O}_{S_{x}} \cdot x^{-1} u \oplus \mathcal{O}_{S_{x}}$ and $\left.\mathcal{F}_{1}\right|_{S_{y}} \cong \mathcal{O}_{S_{y}} \cdot y^{-1} v \oplus \mathcal{O}_{S_{y}}$ and transition matrix

$$
M=\left(\begin{array}{cc}
1 & -x^{-1} y^{-1} \\
0 & 1
\end{array}\right) \in \mathrm{GL}_{2}\left(k\left[x^{ \pm 1}, y^{ \pm 1}\right]\right) .
$$

Since $M$ is equal to the product

$$
M=M_{x} \cdot M_{y}=\left(\begin{array}{cc}
x^{-1} & 0 \\
-y & x
\end{array}\right) \cdot\left(\begin{array}{cc}
x & -y^{-1} \\
y & 0
\end{array}\right)
$$

where $M_{x} \in \mathrm{GL}_{2}\left(k\left[x^{ \pm 1}, y\right]\right)$ and $M_{y} \in \mathrm{GL}_{2}\left(k\left[x, y^{ \pm 1}\right]\right)$, we see that $\mathcal{F}_{1}$ is equal to the free sub- $\mathcal{O}_{S}$-module of $\mathcal{A}$ generated by $u$ and $v$, so that $\mathcal{R}\left(\mathrm{SL}_{2}, \mu\right) \cong \mathcal{O}_{S}[u, v]$.

Composing with the structure map $S \rightarrow \operatorname{Spec}(k)$, we view $X=\mathrm{SL}_{2}$ as the normal affine $k$-variety with $\mathbb{G}_{a}$-action associated to the locally nilpotent $k$-derivation $\partial=x \frac{\partial}{\partial u}+y \frac{\partial}{\partial v}$ of its coordinate ring $A=$ $k[x, y, u, v] /(x v-y u-1)$. The associated filtration $\left\{F_{n}\right\}_{n \geq 0}$ is given by $A_{0}=F_{0}=\operatorname{Ker} \partial=k[x, y]$ and

$$
F_{n}=\operatorname{Ker}^{n+1}=\sum_{p+q=n} A_{0} \cdot u^{p} v^{q}+F_{n-1}, \quad n \geq 1 .
$$

The Rees algebra $R(A, \partial)$ is thus equal to the quotient of the polynomial ring $A_{0}[u, v, \theta]$, endowed with the grading given by the weights $\left(\omega_{u}, \omega_{v}, \omega_{\theta}\right)=(1,1,1)$, by the principal homogeneous ideal generated by $x v-y u-\theta$, hence to the polynomial ring $A_{0}[u, v]=\Gamma\left(S, \mathcal{R}\left(\mathrm{SL}_{2}, \mu\right)\right)$.

\section{ExAMPLES AND APPLiCATIONS}

In this section, we first illustrate the computation and geometric properties of Rees algebras on a series of classical examples in the study of additive group actions on affine varieties, with a particular focus on the interplay between the relative and absolute Rees algebras and the construction of vector bundles of rank two on certain geometric quotients. We then consider an application of Rees algebras to the construction of families of affine extensions of $\mathbb{G}_{a}$-torsors over punctured smooth surfaces.

3.1. Danielewski hypersurfaces in $\mathbb{A}_{k}^{3}$. Given a polynomial $P \in k[x, y]$ such that $P(0, y)$ is non-constant, with simple roots, and an integer $n \geq 1$, we let $S_{n, P}$ be the smooth surface in $\mathbb{A}_{k}^{3}=\operatorname{Spec}(k[x, y, z])$ with equation $x^{n} z=P(x, y)$. For every nonzero polynomial $q(x) \in k[x]$, the surface $S_{n, P}$ is equipped with a nontrivial $\mathbb{G}_{a}$-action $\mu$ associated to the locally nilpotent $k[x]$-derivation

$$
q(x) \partial_{n, P}=q(x)\left(x^{n} \frac{\partial}{\partial y}+\frac{\partial P}{\partial y}(x, y) \frac{\partial}{\partial z}\right)
$$

of its coordinate ring $A_{n, P}$. Letting $d=\operatorname{deg}_{y}(P)$, the corresponding ascending filtration of $A_{n, P}$ is given by $A_{0}=F_{0}=k[x]$ and

$$
F_{d n+k}=k[x] \cdot y^{d n+k}+k[x] \cdot z^{n}+F_{d n+k-1} .
$$

Let $k[x][y, \theta, z]$ be endowed with the grading given by the weights $\left(\omega_{x}, \omega_{y}, \omega_{\theta}, \omega_{z}\right)=(0,1,1, d)$ and let $\tilde{P}(x, y, \theta) \in k[x][y, \theta]$ be the unique homogeneous polynomial with respect to the induced grading such that $P(x, y)=\tilde{P}(x, y, 1)$. The Rees algebra $R\left(A_{n, P}, \partial_{n, P}\right)$ is isomorphic to the quotient of $A_{0}[y, \theta, z]$ by the principal homogeneous ideal generated by $x^{n} z-\tilde{P}(x, y, \theta)$. So $\operatorname{Proj}_{k}\left(R\left(A_{n, P}, \partial_{n, P}\right)\right)$ is isomorphic to the closed sub-scheme $\bar{S}_{n, P}$ of $\mathbb{A}_{k}^{1} \times \mathbb{P}(1,1, d)=\operatorname{Proj}_{k}\left(A_{0}[\theta, y, z]\right)$ with equation $x^{n} z-\tilde{P}(x, y, \theta)=0$, in which $S_{n, P}$ embeds as the complement of the relative hyperplane section

$$
\{\theta=0\} \cap \bar{S}_{n, P}=\left\{x^{n} z-P(x, y, 0)=0\right\} .
$$

The fiber of $\operatorname{pr}_{x}: \bar{S}_{n, P} \rightarrow \mathbb{A}_{k}^{1}$ over the origin is equal to the union of $\operatorname{deg}(\tilde{P}(0, y, \theta))$ copies of the projective line $\mathbb{P}_{k}^{1}$ all intersecting at the point $[0: 0: 1] \in \mathbb{P}(1,1, d)$.

Since $P(0, y)$ has simple roots, the $\mathbb{G}_{a}$-action associated to $\partial_{n, P}$ is fixed point free and the $\mathbb{G}_{a}$-invariant projection $\operatorname{pr}_{x}: S_{n, P} \rightarrow \mathbb{A}_{k}^{1}$ factors through a $\mathbb{G}_{a}$-torsor $\rho: S_{n, P} \rightarrow \breve{\mathbb{A}}_{k}^{1}$ over the irreducible non-separated curve $\delta: \breve{\mathbb{A}}_{k}^{1} \rightarrow \mathbb{A}_{k}^{1}$ obtained from $\mathbb{A}_{k}^{1}=\operatorname{Spec}(k[x])$ by replacing the origin $\{0\}$ by as many disjoint copies as there are irreducible components in the fiber $\operatorname{pr}_{x}^{-1}(\{0\}) \cong \operatorname{Spec}(k[y, z] /(P(0, y)))[6,12]$. We can thus 
consider the action $\mu$ as being given by a locally nilpotent $\mathcal{O}_{\breve{\mathbb{A}}_{k}^{1}}$-derivation $\breve{\partial}$ of $\rho_{*} \mathcal{O}_{S_{n, P}}$. By Proposition 12 , the Rees algebra $\mathcal{R}\left(S_{n, P}, \mu\right)$ is then canonically isomorphic to the symmetric algebra $\operatorname{Sym}^{\prime} \mathcal{F}_{1}$ of the locally free sheaf $\mathcal{F}_{1}=\mathcal{K}$ er $\breve{\partial}^{2}$ of rank 2 on $\breve{\mathbb{A}}_{k}^{1}$ which fits in the exact sequence

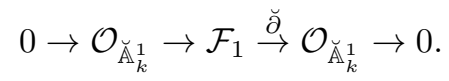

By Lemma 26, we have $R\left(A_{n, P}, \partial_{n, P}\right) \cong \Gamma\left(V, \mathcal{O}_{V}\right)$ where $p: V=\operatorname{Spec}_{\breve{\mathbb{A}}_{k}^{1}}\left(\operatorname{Sym}^{\prime} \mathcal{F}_{1}\right) \rightarrow \breve{\mathbb{A}}_{k}^{1}$ is the vector bundle of rank 2 on $\breve{\mathbb{A}}_{k}^{1}$ determined by $\mathcal{F}_{1}$.

If $\operatorname{deg} P(0, y) \geq 2$, then

$$
R\left(A_{n, P}, \partial_{n, P}\right) \otimes_{A_{0}}\left(A_{0} /(x)\right) \cong k[y, \theta, z] /(\tilde{P}(0, y, \theta))
$$

is not a polynomial ring in two variables over $k=A_{0} /(x)$. So $R\left(A_{n, P}, \partial_{n, P}\right)$ is not isomorphic to a polynomial ring in two variables over $A_{0}$, which implies that $V$ is a nontrivial vector bundle over $\breve{\mathbb{A}}_{k}^{1}$, since otherwise $\Gamma\left(V, \mathcal{O}_{V}\right) \cong R\left(A_{n, P}, \partial_{n, P}\right)$ would be isomorphic to a polynomial ring in two variables over

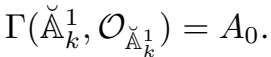

Otherwise, if $\operatorname{deg} P(0, y)=1$, then $\operatorname{pr}_{x}: S_{n, P} \rightarrow \mathbb{A}_{k}^{1}=\operatorname{Spec}\left(A_{0}\right)$ is a $\mathbb{G}_{a}$-torsor for the $\mathbb{G}_{a}$-action determined by the $k$-derivation $\partial_{n, P}$, hence is the trivial one since $\operatorname{Spec}\left(A_{0}\right)$ is affine. It follows in turn that $V$ is the trivial rank 2 vector bundle on $\operatorname{Spec}\left(A_{0}\right)$. In the special case where $P$ is equal to the constant polynomial $y, S_{n, P}$ is isomorphic to $\mathbb{A}_{k}^{2}=\operatorname{Spec}(k[x, z])$ and the $\mathbb{G}_{a}$-action given by $q(x) \partial_{n, P}$ concides with that given by the locally nilpotent $k[x]$-derivation $\partial=q(x) \frac{\partial}{\partial z}$. It is a classical result [27] that every $\mathbb{G}_{a^{-}}$ action on $\mathbb{A}_{k}^{2}$ is conjugate to an action defined by a locally nilpotent derivation of this form. The corresponding filtration is given by $A_{0}=F_{0}=k[x]$ and

$$
\begin{aligned}
& F_{1}=k[x] y \oplus k[x] \cdot 1 \\
& F_{n}=k[x] \cdot y^{n} \oplus F_{n-1} \cong \operatorname{Sym}_{F_{0}}^{n} F_{1}, \quad \text { for } n \geq 2
\end{aligned}
$$

so that we have an isomorphism of graded $k[x]$-algebras $R(k[x, z], \partial) \cong k[x][y, \theta]$, where $\theta$ and $y$ both have homogeneous degree 1 .

3.2. A smooth affine threefold whose geometric quotient is quasi-projective but not quasi-affine. It is known in general that the geometric quotient $X / \mathbb{G}_{a}$ of a proper $\mathbb{G}_{a}$-action on a factorial affine variety is a quasi-affine variety (see e.g. [5]). For smooth affine threefolds $X$, factorial or not, it is a consequence of Chow's Lemma that the algebraic space geometric quotient $X / \mathbb{G}_{a}$ of a proper $\mathbb{G}_{a}$-action is a quasi-projective surface. In this subsection, we consider a simple example of a smooth non-factorial affine threefold $X$ endowed with a proper $\mathbb{G}_{a}$-action, whose geometric quotient is a smooth quasi-projective surface which is not quasi-affine.

Let $S_{0}=\operatorname{Spec}(k[x, y])$ and let $X \subset \mathbb{A}_{S_{0}}^{3}=\operatorname{Spec}\left(k[x, y]\left[w_{1}, w_{2}, w_{3}\right]\right)$ be the smooth threefold defined by the system of equations

$$
\begin{cases}y w_{3}-w_{1} w_{2} & =0 \\ x w_{2}-y\left(y w_{1}+1\right) & =0 \\ x w_{3}-w_{1}\left(y w_{1}+1\right) & =0 .\end{cases}
$$

The threefold $X$ can be endowed with a fixed point free $\mathbb{G}_{a, S_{0}}$-action $\mu: \mathbb{G}_{a, S_{0}} \times_{S_{0}} X \rightarrow X$ induced by the locally nilpotent $k[x, y]$-derivation

$$
\partial=x \frac{\partial}{\partial w_{1}}+y^{2} \frac{\partial}{\partial w_{2}}+\left(2 y w_{1}+1\right) \frac{\partial}{\partial w_{3}}
$$

of its coordinate ring $A$. The ring of invariants $A_{0}=\operatorname{Ker} \partial$ is equal to $k[x, y]$. The Rees algebra $R(A, \partial)$ is isomorphic to the quotient of the polynomial ring $A_{0}\left[\theta, W_{1}, W_{2}, W_{3}\right]$ in four variables over $A_{0}$ with weights $\left(\omega_{\theta}, \omega_{W_{1}}, \omega_{W_{2}}, \omega_{W_{3}}\right)=(1,1,1,2)$ by the homogeneous ideal $I$ generated by the polynomials $x W_{2}-y\left(y W_{1}+\theta\right), y W_{3}-W_{1} W_{2}$ and $x W_{3}-W_{1}\left(y W_{1}+\theta\right) . \operatorname{So~} \operatorname{Proj}_{k}(R(A, \partial))$ is isomorphic to the closed sub-scheme $Y$ of

$$
\mathbb{A}_{k}^{2} \times \mathbb{P}(1,1,1,2)=\operatorname{Proj}_{A_{0}}\left(A_{0}\left[\theta, W_{1}, W_{2}, W_{3}\right]\right)
$$


defined by the vanishing of these polynomials. The threefold $X$ embeds in $Y$ as the complement of the relative hyperplane section $V_{+}(\theta) \cap Y \subset \mathbb{P}(1,1,2)$, defined by the equations $x W_{2}-y^{2} W_{1}=0, y W_{3}-W_{1} W_{2}=0$ and $x W_{3}-y W_{1}^{2}=0$.

It is easily seen from this description that the restriction of $\bar{\pi}=\operatorname{pr}_{\mathbb{A}_{k}^{2}}: Y \rightarrow S_{0}=\mathbb{A}_{k}^{2}$ over the complement of the origin $o=\{(0,0)\}$ is a Zariski locally trivial $\mathbb{P}^{1}$-bundle having $\{\theta=0\}$ as a section, so that the restriction of the algebraic quotient morphism $\pi=\operatorname{pr}_{x, y}: X \rightarrow S_{0}$ over $S_{0} \backslash\{o\}$ is a $\mathbb{G}_{a}$-torsor $P \rightarrow S_{0} \backslash$ $\{o\}$. More explicitly, letting $S_{0, x}=\operatorname{Spec}\left(k\left[x^{ \pm 1}, y\right]\right)$ and $S_{0, y}=\operatorname{Spec}\left(k\left[x, y^{ \pm 1}\right]\right)$, we have $\mathbb{G}_{a}$-equivariant isomorphisms

$$
\begin{cases}\pi^{-1}\left(S_{0, x}\right) \cong \operatorname{Spec}\left(k\left[x^{ \pm 1}, y\right]\left[x^{-1} w_{1}\right]\right) & \cong S_{0, x} \times \mathbb{G}_{a} \\ \pi^{-1}\left(S_{0, y}\right) \cong \operatorname{Spec}\left(k\left[x, y^{ \pm 1}\right]\left[-y^{-2} w_{2}\right]\right) & \cong S_{0, y} \times \mathbb{G}_{a}\end{cases}
$$

where $\mathbb{G}_{a}$ acts on $S_{0, x} \times \mathbb{G}_{a}$ and $S_{0, y} \times \mathbb{G}_{a}$ by translations on the second factor. On the other hand, the scheme-theoretic fiber $\bar{\pi}^{-1}(o) \subset \mathbb{P}(1,1,1,2)$ is the union of the surface $\left\{W_{1}=0\right\} \cong \mathbb{P}(1,1,2)$ and the line $\left\{\theta=W_{2}=0\right\}$. It follows that $\pi^{-1}(o)=\bar{\pi}^{-1}(o) \backslash V_{+}(\theta)$ is isomorphic to $\mathbb{A}_{k}^{2}=\operatorname{Spec}\left(k\left[w_{2}, w_{3}\right]\right)$. In particular the class group of $X$ is isomorphic to $\mathbb{Z}$, generated by the class of the divisor $\pi^{-1}(o)$, and the algebraic quotient morphism $\pi: X \rightarrow S_{0}$ is not the geometric quotient of the fixed point free action $\mu$ on $X$. Let

$$
\tau=\operatorname{pr}_{S_{0}}: S_{1}=\{x v-y u=0\} \subset S_{0} \times \mathbb{P}_{[u: v]}^{1} \rightarrow S_{0}
$$

be the blow-up of the origin $o \in S_{0}$. The morphism $\pi$ lifts to a morphism

$$
\tilde{\pi}: X \rightarrow S_{1}, \quad\left(x, y, w_{1}, w_{2}, w_{3}\right) \mapsto\left((x, y),\left[y w_{1}+1: w_{2}\right]\right) .
$$

which maps $\pi^{-1}(o)$ dominantly onto the exceptional divisor $E \cong \tau^{-1}(o) \cong \operatorname{Proj}(k[u, v])$ of $\tau$.

Lemma 28. The morphism $\tilde{\pi}: X \rightarrow S_{1}$ factors through a Zariski locally trivial $\mathbb{G}_{a}$-torsor $\rho: X \rightarrow S$ over the smooth quasi-projective but not quasi-affine surface $S=S_{1} \backslash\left\{o_{1}\right\}$, where $o_{1}=((0,0),[0: 1]) \in E \subset$ $S_{1}$.

Proof. The induced $\mathbb{G}_{a}$-action on $\pi^{-1}(o) \cong \operatorname{Spec}\left(k\left[w_{2}, w_{3}\right]\right)$ is the translation defined by the locally nilpotent $k$-derivation $\partial_{w_{3}}$ of $k\left[w_{2}, w_{3}\right]$. The morphism $\tilde{\pi}: X \rightarrow S_{1}$ is $\mathbb{G}_{a}$-invariant and the induced morphism

$$
\left.\tilde{\pi}\right|_{\pi^{-1}(o)}: \pi^{-1}(o)=\operatorname{Spec}\left(k\left[w_{2}, w_{3}\right]\right) \rightarrow E, \quad\left(w_{2}, w_{3}\right) \mapsto\left[1: w_{2}\right]
$$

factors as the composition of the geometric quotient $\pi^{-1}(o) \rightarrow \pi^{-1}(o) / \mathbb{G}_{a} \simeq \operatorname{Spec}\left(k\left[w_{2}\right]\right)$ with the open immersion $\pi^{-1}(o) / \mathbb{G}_{a} \hookrightarrow E$ of $\pi^{-1}(o) / \mathbb{G}_{a}$ as the complement of the point $o_{1}=((0,0),[0: 1]) \in E$. It follows that $\tilde{\pi}$ factors through a surjective morphism $\rho: X \rightarrow S=S_{1} \backslash\left\{o_{1}\right\}$ whose fibers all consist of precisely one $\mathbb{G}_{a}$-orbit. Since $\rho$ is a smooth morphism, it is thus a $\mathbb{G}_{a}$-torsor. By construction $S$ is smooth and quasi-projective and $\left.\tau\right|_{S}: S \rightarrow B$ induces an isomorphism $\left(\left.\tau\right|_{S}\right)^{*}: A_{0} \rightarrow \Gamma\left(S, \mathcal{O}_{S}\right)$. If $S$ was quasi-affine, then $\left.\tau\right|_{S}: S \rightarrow S_{0}=\operatorname{Spec}\left(\Gamma\left(S, \mathcal{O}_{S}\right)\right)$ would be an open immersion, which is impossible since $\left.\tau\right|_{S}$ contracts $E \cap S \cong \mathbb{A}_{k}^{1}$ to the point $o \in S_{0}$.

By Proposition 12, the Rees $\mathcal{O}_{S}$-algebra $\mathcal{R}(X, \mu)$ is equal to the symmetric algebra of a Zariski locally free sheaf $\mathcal{F}_{1}$ of rank 2 on $S$. The corresponding rank 2 vector bundle $p: V=\operatorname{Spec}_{S}\left(\operatorname{Sym}^{\prime} \mathcal{F}_{1}\right) \rightarrow S$ is nontrivial. Indeed otherwise $\Gamma\left(V, \mathcal{O}_{V}\right)$ would be isomorphic to a polynomial ring in two variables over $\Gamma\left(S, \mathcal{O}_{S}\right) \cong A_{0}$ but on the other hand it follows from Lemma 26 and the description above that

$$
\Gamma\left(V, \mathcal{O}_{V}\right) \cong R(A, \partial) \cong A_{0}\left[\theta, W_{1}, W_{2}, W_{3}\right] / I
$$

is not a polynomial ring in two variables over $A_{0}$.

3.3. A triangular $\mathbb{G}_{a}$-action on $\mathbb{A}_{k}^{3}$ and the Russell cubic threefold. Let $\mathbb{A}_{k}^{4}=\operatorname{Spec}(k[x, y, z, t])$ be endowed with the $\mathbb{G}_{a}$-action defined by the triangular locally nilpotent $k[x, t]$-derivation

$$
\Delta=x^{2} \frac{\partial}{\partial y}+2 y \frac{\partial}{\partial z} .
$$

The kernel $A_{0}=F_{0}$ of $\Delta$ is equal to $k[x, t, w]$ where $w=x^{2} z-y^{2}$, and for every $n=i+2 j \geq 1$, we have

$$
F_{n}=\operatorname{Ker} \Delta^{n+1}=\sum_{i+2 j=n} A_{0} y^{i} z^{j}+F_{n-1} .
$$


The Rees algebra $R(k[x, y, z, t], \Delta)$ is isomorphic to the quotient of the polynomial ring $A_{0}[y, \theta, z]$, where $y, \theta$ and $z$ have homogenenous degrees 1,1 and 2 respectively, by the homogeneous ideal generated by $x^{2} z-y^{2}-w \theta^{2}$.

The $\mathbb{G}_{a}$-action on $\mathbb{A}_{k}^{4}$ defined by $\Delta$ is fixed point free outside the $\mathbb{G}_{a}$-invariant plane

$$
P=\{x=y=0\} \cong \operatorname{Spec}(k[z, t]) .
$$

The induced $\mathbb{G}_{a}$-action $\mu$ on the quasi-affine fourfold $\mathbb{A}_{k}^{4} \backslash P$ admits a geometric quotient in the category of algebraic spaces in the form of an étale locally trivial $\mathbb{G}_{a}$-torsor $\rho: \mathbb{A}_{k}^{4} \backslash P \rightarrow S$ over an algebraic space $S=\left(\mathbb{A}_{k}^{4} \backslash P\right) / \mathbb{G}_{a}$. The latter is the $\mathbb{A}^{1}$-cylinder $\mathfrak{S} \times \operatorname{Spec}(k[t])$ over a 2-dimensional smooth algebraic space of finite type

$$
\delta: \mathfrak{S} \rightarrow \mathbb{A}_{k}^{2} \backslash\{(0,0)\}=\operatorname{Spec}(k[x, w]) \backslash\{(0,0)\}
$$

obtained from $\mathbb{A}_{k}^{2} \backslash\left\{(0,0\}\right.$ by replacing the curve $\{x=0\} \cong \operatorname{Spec}\left(k\left[w^{ \pm 1}\right]\right)$ by the total space of the étale double cover $\operatorname{pr}_{w}: \operatorname{Spec}\left(k\left[y, w^{ \pm 1}\right] /\left(y^{2}+w\right)\right) \cong \operatorname{Spec}\left(k\left[y^{ \pm 1}\right]\right) \rightarrow \operatorname{Spec}\left(k\left[w^{ \pm 1}\right]\right)$ (see e.g. [7] and the references therein). Let $\left\{\mathcal{F}_{n}\right\}_{n \geq 0}$ be the ascending filtration of $\rho_{*} \mathcal{O}_{\mathbb{A}_{k}^{4} \backslash P}$ associated to the $\mathbb{G}_{a, S^{-}}$ action $\mu$. Since $\rho: \mathbb{A}_{k}^{4} \backslash P \rightarrow S$ is a $\mathbb{G}_{a, S}$-torsor, it follows from Proposition 12 that the Rees $\mathcal{O}_{S^{-}}$ algebra $\mathcal{R}\left(\mathbb{A}_{k}^{4} \backslash P, \mu\right)$ is equal to the symmetric algebra of the rank 2 étale locally free sheaf $\mathcal{F}_{1}$ on $S$. Let $p: V=\operatorname{Spec}_{S}\left(\operatorname{Sym}^{\prime} \mathcal{F}_{1}\right) \rightarrow S$ be the corresponding vector bundle. Since $P$ has pure codimension 2 in $\mathbb{A}_{k}^{4}$, we have $\Gamma\left(V, \mathcal{O}_{V}\right)=R(k[x, y, z, t], \Delta)$ by Lemma 26 . Since $R(k[x, y, z, t], \Delta)$ is not isomorphic to a polynomial ring in two variables over $\Gamma\left(S, \mathcal{O}_{S}\right)=A_{0}$, it follows that $p: V \rightarrow S$ is a nontrivial vector bundle.

The closed subsets $X_{1}$ and $X_{3}$ of $\mathbb{A}_{k}^{4}$ with equations $x^{2} z=y^{2}+t$ and $x^{2} z=y^{2}+t^{3}+x$ are $\mathbb{G}_{a}$-invariant, respectively isomorphic to $\mathbb{A}_{k}^{3}=\operatorname{Spec}(k[x, y, z])$ and the Russell cubic threefold [21]. The restrictions of $\rho: \mathbb{A}_{k}^{4} \backslash P \rightarrow S$ to $X_{1} \backslash P=\operatorname{Spec}(k[x, y, z]) \backslash\{x=y=0\}$ and $X_{3} \backslash P=X_{3} \backslash\{x=y=t=0\}$ are $\mathbb{G}_{a}$-torsors over the closed subspaces $S_{1}$ and $S_{3}$ of $S$ whose ideal sheaves are generated by $w-t$ and $w-\left(t^{3}+x\right)$ respectively. These two spaces are isomorphic to $\mathfrak{S}$ [8, Lemma 3.2], so that $\mathbb{A}_{k}^{3} \backslash\{x=y=0\}$ and $X_{3} \backslash\{x=y=t=0\}$ are étale locally trivial $\mathbb{G}_{a}$-torsors over the same space $\mathfrak{S}$. The Rees algebras for the induced $\mathbb{G}_{a}$-actions on $X_{1}$ and $X_{3}$ are isomorphic to the quotients of $R(k[x, y, z, t], \Delta)$ by the homogeneous ideals generated by $w-t$ and $w-\left(t^{3}+x\right)$ respectively, hence to

$$
R_{1}=k[x, t][y, \theta, z] /\left(x^{2} z-y^{2}-t \theta^{2}\right) \quad \text { and } \quad R_{3}=k[x, t][y, \theta, z] /\left(x^{2} z-y^{2}-\left(t^{3}+x\right) \theta^{2}\right)
$$

respectively. Since these are not polynomial rings in two variables over

$$
\Gamma\left(\mathfrak{S}, \mathcal{O}_{\mathfrak{S}}\right) \cong k[x, t]=\Gamma\left(X_{1}, \mathcal{O}_{X_{1}}\right)^{\mathbb{G}_{a}}=\Gamma\left(X_{3}, \mathcal{O}_{X_{3}}\right)^{\mathbb{G}_{a}},
$$

it follows that the restrictions $V_{1}$ and $V_{3}$ of $V$ to $S_{1} \cong \mathfrak{S}$ and $S_{3} \cong \mathfrak{S}$ are both nontrivial vector bundles of rank 2.

Lemma 29. The vector bundles $p_{1}: V_{1} \rightarrow \mathfrak{S}$ and $p_{3}: V_{3} \rightarrow \mathfrak{S}$ are not isomorphic.

Proof. Indeed, if $V_{1}$ and $V_{3}$ were isomorphic as vector bundles then the graded $\Gamma\left(\mathfrak{S}, \mathcal{O}_{\mathfrak{S}}\right)$-algebras

$$
\Gamma\left(V_{1}, \mathcal{O}_{V_{1}}\right) \cong \Gamma\left(\mathfrak{S}, p_{1 *} \mathcal{O}_{V_{1}}\right) \text { and } \Gamma\left(V_{3}, \mathcal{O}_{V_{3}}\right) \cong \Gamma\left(\mathfrak{S}, p_{3 *} \mathcal{O}_{V_{3}}\right)
$$

would be isomorphic. Combined with Lemma 26, this would imply that $R_{1}$ and $R_{3}$ are isomorphic graded $k[x, t]$-algebras, hence that $k[x, y, z]=\Gamma\left(X_{1}, \mathcal{O}_{X_{1}}\right) \cong R_{1} /(1-\theta) R_{1}$ and $\Gamma\left(X_{3}, \mathcal{O}_{X_{3}}\right) \cong R_{3} /(1-\theta) R_{3}$ were isomorphic, contradicting the fact the Russel cubic $X_{3}$ is not isomorphic to $\mathbb{A}_{k}^{3}$ [21].

3.4. Winkelmann's proper locally trivial action on $\mathbb{A}_{k}^{5}$. A $\mathbb{G}_{a}$-action on an affine space $\mathbb{A}_{k}^{n}$ is called a translation if its geometric quotient $\mathbb{A}_{k}^{n} / \mathbb{G}_{a}$ is isomorphic to $\mathbb{A}_{k}^{n-1}$ and $\mathbb{A}_{k}^{n}$ is equivariantly isomorphic to $\left(\mathbb{A}_{k}^{n} / \mathbb{G}_{a}\right) \times \mathbb{G}_{a}$ on which $\mathbb{G}_{a}$ acts by translations on the second factor. It is classical that proper $\mathbb{G}_{a}$-actions on $\mathbb{A}_{k}^{2}$ and $\mathbb{A}_{k}^{3}$ are translations. The question whether a proper $\mathbb{G}_{a}$-action on $\mathbb{A}_{k}^{4}$ is a translation is still widely open (see e.g. $[10,20]$ for partial results). Examples of proper $\mathbb{G}_{a}$-actions on affine spaces $\mathbb{A}_{k}^{n}, n \geq 5$, which fail to be translations were constructed by Winkelmann [30]. We consider the simplest of these examples, in dimension 5 . 
Let $A=k[u, v][x, y, z]$ and let $\mu$ be the fixed point free $\mathbb{G}_{a}$-action on $\mathbb{A}_{k}^{5}=\operatorname{Spec}(A)$ determined by the triangular locally nilpotent $k[u, v]$-derivation

$$
\partial=u \frac{\partial}{\partial x}+v \frac{\partial}{\partial y}+(1+w) \frac{\partial}{\partial z}
$$

where $w=x v-y u$.

Lemma 30. The Rees algebra $R(A, \partial)$ is isomorphic to the quotient of the polynomial ring $A\left[w, c_{1}, c_{2}, \theta\right]$ endowed with the grading defined by the weights $\omega_{u}=\omega_{v}=\omega_{w}=\omega_{c_{1}}=\omega_{c_{2}}=0, \omega_{x}=\omega_{y}=\omega_{z}=$ $\omega_{\theta}=1$ by the homogeneous ideal I generated by the polynomials $v c_{1}-u c_{2}-w(w+1), c_{2} x-c_{1} y+w z$, $w \theta+u y-v x, c_{2} \theta+v z-(1+w) y$ and $c_{1} \theta+u z-(1+w) x$.

Proof. By [30], the kernel $A_{0}=F_{0}$ of $\partial$ is generated by $u, v, w=x v-y u, c_{1}=x(1+w)-u z$ and $c_{2}=y(1+w)-v z$, with the unique relation $v c_{1}-u c_{2}-w(1+w)=0$. Furthermore, since we have

$$
F_{1}=F_{0} \cdot x+F_{0} \cdot y+F_{0} \cdot z+F_{0},
$$

the Rees algebra $R(A, \partial)$ is generated over $A_{0}$ by $x, y, z$ and $\theta$. These elements of $A$ satisfy the linear dependence relations $c_{1} \theta+u z-(1+w) x=0, c_{2} \theta+v z-(1+w) y=0$ and $w \theta+u y-v x=0$ over $A_{0}$. Furthermore, the element $c_{2} x-c_{1} y+w z \in F_{1}$ belongs to $F_{0}$, which yields the additional relation $c_{2} x-c_{1} y+w z=0 \cdot \theta=0$. It follows that $R(A, \partial)$ is a quotient of the ring $B=k\left[u, v, w, c_{1}, c_{2}\right][x, y, z, \theta] / I$. The images of $w$ and $(1+w)$ generate the unit ideal in $B$, and the localizations $B_{(1+w)} \cong A_{0}\left[(1+w)^{-1}\right][z, \theta]$ and $B_{w} \cong A_{0}\left[w^{-1}\right][x, y]$ are integral domains of dimension 6 . The homomorphism $B \rightarrow B_{w} \times B_{(1+w)}$ is thus injective, which implies in turn that $B$ is an integral domain of dimension 6 . Since $R(A, \partial)$ is itself an integral domain of dimension 6 as $A$ is of dimension 5 , we conclude that $R(A, \partial)=B$.

The image of the algebraic quotient morphism $\pi: \mathbb{A}_{k}^{5} \rightarrow Q=\operatorname{Spec}\left(A_{0}\right)$ is equal to the complement of the codimension 2 closed subset $W=\{u=v=1+w=0\} \cong \operatorname{Spec}\left(k\left[c_{1}, c_{2}\right]\right)$ in the smooth affine quadric

$$
Q=\left\{v c_{1}-u c_{2}-w(1+w)=0\right\} \subset \mathbb{A}_{k}^{5} .
$$

Furthermore, the corestriction $\rho: \mathbb{A}_{k}^{5} \rightarrow S=Q \backslash W$ of $\pi$ is a $\mathbb{G}_{a}$-torsor, whose class in $H^{1}\left(S, \mathcal{O}_{S}\right)$ is represented by the Čech 1-cocycle

$$
\left\{\frac{c_{1}}{u(1+w)},-\frac{c_{2}}{v(1+w)}, \frac{w}{u v}\right\} \in C^{1}\left(\mathcal{U}, \mathcal{O}_{S}\right)
$$

on the covering $\mathcal{U}$ of $S$ by the principal affine open subsets $S_{u}, S_{v}$ and $S_{1+w}$. Viewing $\mathbb{A}_{k}^{5}$ as a $\mathbb{G}_{a}$-torsor over $S$, it follows from Proposition 12 that the quasi-coherent $\mathcal{O}_{S}$-algebra $\mathcal{R}\left(\mathbb{A}_{k}^{5}, \mu\right)$ is isomorphic to the symmetric algebra Sym $\mathcal{F}_{1}$ of the rank 2 locally free sheaf $\mathcal{F}_{1}=\mathcal{K} e r \delta_{S}^{2}$, where $\delta_{S}$ denotes the $\mathcal{O}_{S}$-derivation of $\rho_{*} \mathcal{O}_{\mathbb{A}_{k}^{5}}$ induced by $\partial$. Let $p: V=\operatorname{Spec}_{S}\left(\operatorname{Sym}^{\prime} \mathcal{F}_{1}\right) \rightarrow S$ be the corresponding vector bundle.

It follows from the proof of Lemma 30 that the morphism $\bar{p}: Y=\operatorname{Spec}(R(A, \partial)) \rightarrow Q$ induced by the inclusion $A_{0}=\Gamma\left(Q, \mathcal{O}_{Q}\right) \subset R(A, \partial)$ is vector bundle of rank 2, which becomes trivial on the cover of $Q$ by the the principal affine open subsets $Q_{w}$ and $Q_{(1+w)}$, and whose restriction over $S=Q \backslash W$ coincides with the vector bundle $p: V=\operatorname{Spec}_{S}\left(\operatorname{Sym}^{\prime} \mathcal{F}_{1}\right) \rightarrow S$.

Lemma 31. The vector bundles $\bar{p}: Y=\operatorname{Spec}(R(A, \partial)) \rightarrow Q$ and $p: V=\operatorname{Spec}_{S}\left(\operatorname{Sym}^{\cdot} \mathcal{F}_{1}\right) \rightarrow S$ are nontrivial.

Proof. The homomorphism $A_{0}[x, y, z, \theta] \rightarrow A_{0},(x, y, z, \theta) \mapsto(u, v,(1+w), 0)$ induces a homomorphism $R(A, \partial) \rightarrow A_{0}$ defining a section $s: Q \rightarrow Y$ of $\bar{p}: Y \rightarrow Q$ whose zero locus is equal to the closed variety $W$. Since $W$ is not a scheme-theoretic complete intersection in $Q$ (see e.g. [29, Lemma 6.3]), it follows that $\bar{p}: Y \rightarrow Q$ is a nontrivial vector bundle. In particular $R(A, \partial)$ is not isomorphic to a polynomial ring in two variables over $A_{0}$. This implies that $p: V \rightarrow S$ is a nontrivial vector bundle. Indeed, otherwise, since $W$ has pure codimension 2 in the smooth affine variety $Q, V$ would extend to the trivial vector bundle on $Q$, and then $\Gamma\left(V, \mathcal{O}_{V}\right)=R(A, \partial)$ would be isomorphic to a polynomial ring in two variables over $A_{0}$. 
3.5. Extensions of $\mathbb{G}_{a}$-torsors over punctured surfaces. In this subsection, we present an application of Rees algebras to the construction of affine extensions of $\mathbb{G}_{a}$-torsors over punctured surfaces. The following notion was introduced in $[11,18]$.

Definition 32. Let $\left(S^{\prime}, o\right)$ be a pair consisting of the spectrum of a regular local ring $B$ essentially of finite type and dimension 2 over an algebraically closed field $k$ of characteristic zero and its closed point. A normal affine $\mathbb{G}_{a}$-extension of a nontrivial $\mathbb{G}_{a}$-torsor $\rho: P \rightarrow S=S^{\prime} \backslash\{o\}$ is a $\mathbb{G}_{a}$-equivariant open embedding $j: P \hookrightarrow X$ into an integral normal $k$-scheme $X$ equipped with a surjective affine morphism $\pi: X \rightarrow S^{\prime}$ of finite type and a $\mathbb{G}_{a, S}$-action, such that the commutative diagram

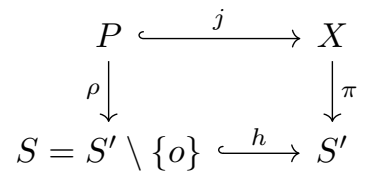

is cartesian, where $h: S \rightarrow S^{\prime}$ denotes the open inclusion.

Let $\mu_{P}: \mathbb{G}_{a, S} \times_{S} P \rightarrow P$ be the $\mathbb{G}_{a, S}$-action on $P$ with corresponding locally nilpotent $\mathcal{O}_{S}$-derivation $\partial_{P}$ of $\rho_{*} \mathcal{O}_{P}$. It follows from Example 14 (see also Example 27) that the Rees algebra $\mathcal{R}(P, \mu)=\bigoplus_{n \geq 0} \mathcal{F}_{n}$ is isomorphic to the polynomial ring algebra $\mathcal{O}_{S}[u, v]$ where $u$ and $v$ are homogeneous variables of degree 1. Furthermore, by Proposition 12 a), the associated homogeneous $\mathcal{O}_{S}$-derivation $\mathcal{R}\left(\partial_{P}\right)$ of degree -1 of $\mathcal{R}(P, \mu)$ is equal to $x \frac{\partial}{\partial u}+y \frac{\partial}{\partial v}$ for some $x, y \in \Gamma\left(S, \mathcal{O}_{S}\right)=B$ such that $(x, y) \mathcal{O}_{S}=\mathcal{O}_{S}$. By Example 14, the nontriviality of $\rho: P \rightarrow S$ is equivalent to the property that the radical of $(x, y) B$ is equal to the maximal ideal $\mathfrak{m}$ of $B$. The image of the element $\theta \in \mathcal{F}_{1}$ in $\mathcal{O}_{S}[u, v]$ is then equal to $x v-y u$, and $P$ is isomorphic to the closed subscheme of $\operatorname{Spec}(B[u, v])$ defined by the equation $\theta=1$.

Given a normal affine extension $j: P \hookrightarrow X=\operatorname{Spec}(A)$, where $A$ is a $B$-algebra of finite type, the $\mathbb{G}_{a, S}$-action on $X$ is determined by a locally nilpotent $B$-derivation $\partial_{X}$ of $A$. We denote by $\left\{F_{X, n}\right\}_{n \geq 0}$ the corresponding filtration of $A$ by its $B$-submodules and by $R\left(A, \partial_{X}\right)=\bigoplus_{n>0} F_{X, n}$ the associated Rees $B$-algebra.

Proposition 33. There is one-to-one correspondence between:

1) Normal affine $\mathbb{G}_{a}$-extensions $j: P \hookrightarrow X=\operatorname{Spec}(A)$ whose Rees algebras $R\left(A, \partial_{X}\right)$ are finitely generated over $B$.

2) Normal finitely generated proper graded B-subalgebras $R_{X}=\bigoplus_{n \geq 0} G_{n}$ of $B[u, v]$ which are stable under the derivation $x \frac{\partial}{\partial u}+y \frac{\partial}{\partial u}$, containing $\theta=x v-y u$ and such that $\left.\tilde{\tilde{G}}_{1}\right|_{S}=\mathcal{F}_{1}$, where $\tilde{G}_{1}$ denotes the sheaf of $\mathcal{O}_{S^{\prime}}$-modules associated to the B-module $G_{1}$.

Proof. Given a normal affine extension $j: P \hookrightarrow X$ of $\rho: P \rightarrow S$, the commutativity of the diagram in the definition implies that we have an injective homomorphism

$$
j^{*}: \pi_{*} \mathcal{O}_{X} \rightarrow \pi_{*} j_{*} \mathcal{O}_{P}=h_{*} \rho_{*} \mathcal{O}_{P} .
$$

Let $\mu_{X}: \mathbb{G}_{a, S^{\prime}} \times_{S^{\prime}} X \rightarrow X$ be the $\mathbb{G}_{a, S^{\prime}}$-action on $X$ and let $\left\{\mathcal{F}_{X, n}\right\}_{n \geq 0}$ be the corresponding ascending filtration of the $\mathcal{O}_{S^{\prime}}$-algebra $\mathcal{A}=\pi_{*} \mathcal{O}_{X}$. The open embedding $j$ being by definition $\mathbb{G}_{a}$-equivariant with respect to the morphism $\tilde{h}: \mathbb{G}_{a, S} \rightarrow \mathbb{G}_{a, S^{\prime}}$ induced by the open inclusion $h: S \rightarrow S^{\prime}$, it follows from Proposition 11 that $j^{*}$ induces an injective homomorphism of graded $\mathcal{O}_{S^{\prime}}$-algebras

$$
\mathcal{R}\left(j^{*}\right): \mathcal{R}\left(X, \mu_{X}\right)=\bigoplus_{n \geq 0} \mathcal{F}_{X, n} \rightarrow h_{*} \mathcal{R}\left(P, \mu_{P}\right)=\bigoplus_{n \geq 0} h_{*} \mathcal{F}_{n} \cong h_{*} \mathcal{O}_{S}[u, v]=\mathcal{O}_{S^{\prime}}[u, v]
$$

which is equivariant with respect to the associated homogeneous $\mathcal{O}_{S^{\prime}}$-derivations $\mathcal{R}\left(\partial_{X}\right)$ and $h_{*} \mathcal{R}\left(\partial_{P}\right)$. Furthermore, since by definition of an affine extension the open embedding $j$ restricts to an equivariant isomomorphism over $S$, it follows that $\mathcal{R}\left(j^{*}\right)$ restricts to an equivariant isomorphism over $S$. Taking global sections over $S^{\prime}$, we obtain an injective homomorphism of graded $B$-algebras

$$
\Gamma\left(S^{\prime}, \mathcal{R}\left(j^{*}\right)\right): \Gamma\left(S^{\prime}, \mathcal{R}\left(X, \mu_{X}\right)\right) \rightarrow \Gamma\left(S^{\prime}, \mathcal{O}_{S^{\prime}}[u, v]\right)=B[u, v],
$$


which is equivariant with respect to the locally nilpotent $B$-derivations $\mathcal{R}\left(\partial_{X}\right)$ and $\Gamma\left(h_{*} \mathcal{R}\left(\partial_{P}\right)\right)=x \frac{\partial}{\partial u}+$ $y \frac{\partial}{\partial v}$. Since $o$ has codimension 2 in the regular scheme $S^{\prime}$, we have $\Gamma\left(S^{\prime}, \mathcal{R}\left(X, \mu_{X}\right)\right)=R\left(A, \partial_{X}\right)$ and $\mathcal{R}\left(X, \mu_{X}\right)=R \widetilde{\left(A, \partial_{X}\right)}$. Since $A$ is normal, so is $R\left(A, \partial_{X}\right)$ by Lemma 18 . Since $\mathcal{R}\left(j^{*}\right)$ maps the constant section $1 \in \Gamma\left(S^{\prime}, \mathcal{O}_{S^{\prime}}\right)$ viewed in $\mathcal{F}_{X, 1}$ to the same section viewed in $h_{*} \mathcal{F}_{1}$, it follows from the definition of $\theta$ (see (1.2)) that $x v-y u \in R\left(A, \partial_{X}\right)$. If the inclusion $R\left(A, \partial_{X}\right) \subset B[u, v]$ is an equality, then

$$
A \cong R\left(A, \partial_{X}\right) /(1-\theta) R\left(A, \partial_{X}\right)=B[u, v] /(1-\theta) B[u, v] \cong \Gamma\left(P, \mathcal{O}_{P}\right),
$$

which contradicts the fact that $j: P \hookrightarrow X$ is an affine extension. Since by Proposition $12 \mathrm{~b}), \mathcal{R}\left(P, \mu_{P}\right) \cong$ Sym $\mathcal{F}_{1}$, the equality $\left.R \widetilde{\left(A, \partial_{X}\right)}\right|_{S}=\mathcal{R}\left(P, \mu_{P}\right)$ is equivalent to the fact that $\left.\widetilde{F}_{X, 1}\right|_{S}=\left.\mathcal{F}_{X, 1}\right|_{S}=\mathcal{F}_{1}$. Summing up, independently of whether $R\left(A, \partial_{X}\right)$ is finitely generated over $B$ or not, $R\left(A, \partial_{X}\right) \subset B[u, v]$ is an integrally closed proper graded $B$-subalgebra of $B[u, v]$, stable under the derivation $x \frac{\partial}{\partial u}+y \frac{\partial}{\partial u}$, containing $\theta=x v-y u$ and such that $\left.\widetilde{F}_{X, 1}\right|_{S}$.

Conversely, given a finitely generated $B$-subalgebra $R_{X}=\bigoplus_{n>0} G_{n}$ of $B[u, v]$ satisfying all these properties, the quotient $A=R_{X} /(1-\theta) R_{X}$ is a finitely generated $B$-subalgebra of $B[u, v] /(1-\theta) B[u, v] \cong$ $\Gamma\left(P, \mathcal{O}_{P}\right)$, stable under the derivation $\partial_{P}$ and such that for the induced locally nilpotent $B$-derivation $\partial_{X}=$ $\left.\partial_{P}\right|_{A}$, we have $R\left(A, \partial_{X}\right) \cong R_{X}$. By Lemma 15 , we have $R_{X}\left[\theta^{-1}\right] \cong A\left[\theta^{ \pm 1}\right]$, so that $A$ is normal as $R_{X}$ is normal by assumption. The affine $S^{\prime}$-scheme $\pi: X=\operatorname{Spec}(A) \rightarrow S^{\prime}$ is thus normal and of finite type, and the morphism $j: P \rightarrow X$ is equivariant. Since $\left.\tilde{G}_{1}\right|_{S}=\mathcal{F}_{1}$, it follows that the restriction of $j$ over $S$ is an isomorphism, so that $j$ is an open embedding of $P$ with complement equal to $\pi^{-1}(o)$. Finally, the inclusion $A \subset \Gamma\left(P, \mathcal{O}_{P}\right)$ is strict since otherwise we would have $R_{X}=B[u, v]$. It follows that $\pi^{-1}(o)$ is not empty, hence that $j: P \hookrightarrow X$ is a normal affine extension of $P$ with finitely generated Rees $B$-algebra $R\left(A, \partial_{X}\right) \cong R_{X}$.

Example 34. The graded proper $B$-subalgebra

$$
R=B[\theta, x u, x v, y v]=\bigoplus_{n \geq 0} G_{n}
$$

of $B[u, v]$ is generated in degree 1 , stable under the derivation $R\left(\partial_{P}\right)=x \frac{\partial}{\partial u}+y \frac{\partial}{\partial v}$ and satisfies $\left.\tilde{G}_{1}\right|_{S} \cong$ $\mathcal{O}_{S}^{\oplus 2}=\left.\mathcal{F}_{1}\right|_{S}$. Writing $X=x u, Z=x v$ and $Y=y v$, we have $R \cong B[\theta, X, Z, Y] / J$ where $J$ is the homogeneous ideal generated by

$$
x Y-y Z, y X-x(Z-\theta), X Y-Z(Z-\theta) .
$$

We thus have

$$
R /(1-\theta) R \cong B[X, Z, Y] /(x Y-y Z, y X-x(Z-1), X Y-Z(Z-1))
$$

which is easily seen to be smooth by the Jacobian criterion. This implies in turn by Lemma 18 that $R$ is normal. The induced $\mathbb{G}_{a, S^{\prime}}$-action on $V=\operatorname{Spec}(R /(1-\theta) R)$ is given by the locally nilpotent $B$-derivation

$$
\partial_{V}=x^{2} \frac{\partial}{\partial X}+y^{2} \frac{\partial}{\partial Y}+x y \frac{\partial}{\partial Z} \text {. }
$$

The open embedding $P \hookrightarrow V$ is given by $(u, v) \mapsto(X, Y, Z)=(x u, y v, x v)$ and the fiber of $\pi: V \rightarrow S^{\prime}$ over the closed point $o$ is isomorphic to the smooth surface with equation $X Y-Z(Z-1)=0$ in $\mathbb{A}_{\kappa}^{3}=$ $\operatorname{Spec}(B / \mathfrak{m} B[X, Y, Z])$, on which the $\mathbb{G}_{a, S^{\prime}}$-action on $V$ restricts to the trivial $\mathbb{G}_{a, \kappa}$-action.

Example 35. (See [11, § 3.4.1]) For every integer $\ell \geq 0$, we let $R_{\ell}$ be the proper graded $B$-subalgebra

$$
R_{\ell}=B\left[\theta, v, x u, u v, x u^{2}, \ldots, x u^{\ell+4}\right]=\bigoplus_{n \geq 0} G_{n}
$$

of $B[u, v]$. It is straightforward to see that $\left.\tilde{G}_{1}\right|_{S} \cong \mathcal{O}_{S}^{\oplus 2}=\left.\mathcal{F}_{1}\right|_{S}$. Furthermore, since $R\left(\partial_{P}\right)\left(x u^{m}\right)=$ $m x^{2} u^{m-1} \in R_{\ell}$ for every $m=1, \ldots, \ell$ and $R\left(\partial_{P}\right)(u v)=x v+y u=-\theta+2 x v \in R_{\ell}$, we get that $R_{\ell}$ is $R\left(\partial_{P}\right)$-stable. The open embedding

$$
P \hookrightarrow V_{\ell}=\operatorname{Spec}\left(R_{\ell} /(1-\theta) R_{\ell}\right)
$$


is given by $(u, v) \mapsto\left(v, x u, u v, x u^{2}, \ldots, x u^{\ell+4}\right)$ and the fiber of $\pi_{\ell}: V_{\ell} \rightarrow S^{\prime}$ over the closed point $o$ of $S^{\prime}$ is isomorphic to $\mathbb{A}_{\kappa}^{2}=\operatorname{Spec}(\kappa[v, u v])$, where $\kappa=B / \mathfrak{m} B$, on which the induced $\mathbb{G}_{a, S^{\prime}}$-action on $V_{\ell}$ restricts to the free $\mathbb{G}_{a, \kappa}$-action $t \cdot(v, u v) \mapsto(v, u v-t)$. Denoting $y \in \mathfrak{m} B$ by $y_{0}, V_{\ell}$ endowed with the $\mathbb{G}_{a, S^{\prime}}$-action induced by $R\left(\partial_{P}\right)$ is equivarianly isomorphic to the smooth subvariety in $S^{\prime} \times_{\mathbb{Z}} \mathbb{A}_{\mathbb{Z}}^{n+2}=$ $\operatorname{Spec}\left(B\left[z_{1}, z_{2}, y_{1}, \ldots, y_{n}\right]\right)$ defined by the system of equations

$$
\begin{cases}y_{i} y_{j}-y_{k} y_{\ell} & =0 \quad i, j, k, \ell=0, \ldots, n, i+j=k+\ell \\ z_{2} y_{i}-z_{1} y_{i+1} & =0 \quad i=0, \ldots, n-1 \\ x y_{i+1}-y_{i}\left(y_{0} z_{1}+1\right) & =0 \quad i=0, \ldots, n-1 \\ x z_{2}-z_{1}\left(y_{0} z_{1}+1\right) & =0,\end{cases}
$$

endowed with the $\mathbb{G}_{a, S^{\prime}}$-action induced by the locally nilpotent $B$-derivation

$$
x \partial_{z_{1}}+\left(2 y_{0} z_{1}+1\right) \partial_{z_{2}}+\sum_{i=1}^{n} i y_{0} y_{i-1} \partial_{y_{i}}
$$

of its coordinate ring. Since $V_{\ell}$ is smooth, hence normal, it follows that $R_{\ell}$ is also normal by Lemma 18 .

\section{REFERENCES}

1. B. Alhajjar, LND-Filtrations and Semi-Rigid Domains,arXiv:1501.00445.

2. B. Alhajjar, Methods to compute ring invariants and applications: a new class of exotic threefolds, arXiv:1506.08522.

3. I. Arzhantsev, U. Derenthal, J. Hausen and A. Laface, Cox rings, Cambridge Studies in Advanced Mathematics, 144. Cambridge University Press, Cambridge, 2015.

4. N. Bourbaki, Commutative algebra, Chapters 1-7. Translated from the French. Reprint of the 1972 edition. Elements of Mathematics (Berlin). Springer-Verlag, Berlin, 1989.

5. J. K. Deveney, D. R. Finston and M. Gehrke, $\mathbb{G}_{a}$-actions on $\mathbb{C}^{n}$, Comm. Alg. 22 (1994) 4977-4988.

6. A. Dubouloz, Danielewski-Fieseler surfaces, Transformation Groups vol. 10, no. 2, (2005), p. 139-162.

7. A. Dubouloz, Exotic $\mathbb{G}_{a}$-quotients of $\mathrm{SL}_{2} \times \mathbb{A}^{1}$, arXiv: 1902.00372 .

8. A. Dubouloz and J. Fasel, Families of $\mathbb{A}^{1}$-contractible affine threefolds, Algebr. Geom., 5(1):1-14, 2018.

9. A. Dubouloz and D. R. Finston, On exotic affine 3-spheres, J. Algebraic Geom. 23 (2014), no. 3, 445-469.

10. A. Dubouloz, D. R. Finston and I. Jaradat, Proper triangular $\mathbb{G}_{a}$-actions on $\mathbb{A}^{4}$ are translations, Algebra Number Theory 8:8 (2014), 1959-1984.

11. A. Dubouloz, I. Hedén, and T. Kishimoto, Equivariant extensions of $\mathbb{G}_{a}$-torsors over punctured surfaces, Ann. Sc. Norm. Super. Pisa Cl. Sci. (To appear), DOI 10.2422/2036-2145.201710_002, arXiv:1707.08768.

12. A. Dubouloz and P.-M. Poloni, On a class of Danielewski surfaces in affine 3-space, Journal of Algebra 321 (2009), p.1797-1812.

13. A. van den Essen, Polynomial automorphisms and the Jacobian conjecture, Progress in Mathematics, 190. Birkhäuser Verlag, Basel, 2000.

14. G. Freudenburg, Canonical factorization of the quotient morphism for an affine $\mathbb{G}_{a}$-variety, Transformation Groups (2018). , arXiv:1602.08786.

15. G. Freudenburg, Algebraic theory of locally nilpotent derivations, Second edition,Encyclopaedia of Mathematical Sciences, 136. Invariant Theory and Algebraic Transformation Groups, VII. Springer-Verlag, Berlin, 2017.

16. A. Grothendieck, Éléments de géométrie algébrique : II. Étude globale élémentaire de quelques classes de morphismes, Publ. Math. I.H.É.S., Tome 8 (1961), 5-222.

17. R. Hartshorne, Algebraic Geometry Graduate Texts in Mathematics Series Volume 52, Springer-Verlag New York, 1977.

18. I. Hedén, Affine extensions of principal additive bundles over a punctured surface, Transform. Groups 21 (2),(2016), 427-449.

19. G. Horrocks, Vector bundles on the punctured spectrum of a local ring, Proc. London Math. Soc. (3) 14 1964, 689-713.

20. S. Kaliman, Proper $\mathbb{G}_{a}$-actions on $\mathbb{C}^{4}$ preserving a coordinate, Algebra Number Theory Volume 12, Number 2 (2018), 227-258.

21. S. Kaliman and L. Makar-Limanov, On the Russell-Koras contractible threefolds, J. Algebraic Geom., 6 no. 2 (1997), 247-268.

22. S. Kaliman and L. Makar-Limanov, AK-invariant of affine domains, Affine Algebraic Geometry, 231-255, Osaka University Press, (2007).

23. S. Kaliman and M. Zaidenberg, Affine modifications and affine hypersurfaces with a very transitive automorphism group, Transform. Groups 4 (1999), no. 1, 53-95.

24. R. Lazarsfeld, Positivity in algebraic geometry I, Ergebnisse der Mathematik und ihrer Grenzgebiete. 3. Folge. 48. Springer-Verlag, Berlin, 2004.

25. G. Laumon et L. Moret-Bailly, Champs Algébriques, Ergebnisse der Mathematik und ihrer Grenzgebiete 39. Springer-Verlag, New York, 2000

26. M. Miyanishi, A remark on an iterative infinite higher derivation, J. Math. Kyoto Univ. 8 (1968) 411-415.

27. R. Rentschler, Opérations du groupe additif sur le plan affine, C. R. Acad. Sci. Paris Sér. A-B 267 (1968) 384-387.

28. C.S. Seshadri, Triviality of vector bundles over the affine space $K^{2}$, Proc. Nat. Acad. Sci. U.S.A. 44 1958, 456-458. 
29. V. Srinivas, Some applications of algebraic cycles to affine algebraic geometry, Algebraic cycles, sheaves, shtukas, and moduli, 185-215, Trends Math., Birkhäuser, Basel, 2008.

30. J. Winkelmann, On free holomorphic $\mathbb{C}_{+}$-actions on $\mathbb{C}^{n}$ and homogeneous Stein manifolds, Math. Ann. 286 (1990), 593-612.

31. O. Zariski, The theorem of Riemann-Roch for high multiples of an effective divisor on an algebraic surface, Ann. of Math. (2) 76 (1962), 560-615.

IMB UMR5584, CNRS, Univ. Bourgogne FranCHE-COMTÉ, F-21000 Dijon, FranCE.

E-mail address: adrien.dubouloz@u-bourgogne.fr

Mathematics Institute, ZeEman BUILDing, University of WARWick COVEnTRY CV4 7AL, UK.

E-mail address: I. Heden@warwick.ac.uk

Department of Mathematics, Faculty of Science, Saitama University, Saitama 338-8570, Japan

E-mail address: tkishimo@rimath.saitama-u.ac.jp 